\title{
Position-element frequency learning is dissociable from Hebb repetition learning
}

$\operatorname{AUTHOR}(S):$

Nakayama, Masataka; Saito, Satoru

\section{CITATION:}

Nakayama, Masataka ...[et al]. Position-element frequency learning is dissociable from Hebb repetition learning. Journal of Memory and Language 2017, 94: 235-253

ISSUE DATE:

2017-06

URL:

http://hdl.handle.net/2433/265324

\section{RIGHT:}

(c) 2016. This manuscript version is made available under the Creative Commons Attribution-NonCommercialNoDerivatives 4.0 International license.; The full-text file will be made open to the public on 1 June 2019 in accordance with publisher's 'Terms and Conditions for Self-Archiving'.; This is not the published version. Please cite only the published version.この論文は出版社版でありません。引用の際には出版社版をご確認ご利用ください。 
Positional repetition in serial learning

1

Running head: Positional repetition in serial learning

Position-element frequency learning is dissociable from Hebb repetition learning

\author{
Masataka Nakayama ${ }^{1,2,3}$
}

Satoru Saito ${ }^{1}$

1. Graduate School of Education Kyoto University, Yoshida-honmachi, Sakyo-ku, Kyoto 606-

$$
\text { 8501, Japan }
$$

2. Kokoro Research Center Kyoto University, 46 Shimoadachi-chou, Yoshida, Sakyo-ku, Kyoto 606-8501, Japan

3. Department of Psychology Carnegie Mellon University, 5000 Forbes Avenue, Pittsburgh, PA

$$
\text { 15213, USA }
$$

Corresponding Authors:

Masataka Nakayama or Satoru Saito

Graduate School of Education Kyoto University, Yoshida-honmachi, Sakyo-ku, Kyoto 6068501, Japan.

Telephone: +81757533067

Fax: +8175753 3066

E-mail: masataka.nakayama.mn@gmail.com or saito.satoru.2z@kyoto-u.ac.jp

Nakayama, M., \& Saito, S. (2017). Position-element frequency learning is dissociable from Hebb repetition learning. Journal of Memory and Language, 94, 235-253. 
Positional repetition in serial learning $\quad 2$

Position-element frequency learning is dissociable from Hebb repetition learning

\section{Introduction}

Serial order control is fundamental to language processing (Lashley, 1951). On one hand, researchers have extensively studied the mechanisms for serial ordering in short-term memory (STM), leading to the development of various conceptual and computational models (Hurlstone, Hitch, \& Baddeley, 2014). On the other hand, interactions of STM and linguistic long-term memory (LTM) have been shown. Acquired knowledge of language structure contributes to short-term retention of novel, but structure-compatible sequences (e.g., Gathercole, Frankish, Pickering, \& Peaker, 1999; Majerus, Linden, Mulder, Meulemans, \& Peters, 2004; Majerus, Martinez Perez, \& Oberauer, 2012; Tanida, Ueno, Lambon Ralph, \& Saito, 2015; see Botvinick \& Plaut, 2006 for a review). Repeated (short-term) retention of a sequence, in turn, leads to the acquisition of linguistic knowledge; therefore phonological STM serves as a language learning device (Baddeley, Gathercole, \& Papagno, 1998; Burgess \& Hitch, 2006; Gupta \& Tisdale, 2009; Page \& Norris, 2009).

Forms of knowledge are acquired through short-term retention of phonological sequences. One form of knowledge acquired through STM functionality is knowledge for individual sequence indicated by the Hebb repetition effect. The Hebb paradigm (Hebb, 1961) is a serial learning paradigm that has been used as a laboratory analogue of word-form learning (Mosse \& Jarrold, 2008; Szmalec, Duyck, Vandierendonck, Mata, \& Page, 2009; Szmalec, Page, \& Duyck, 2012). In a typical experiment, participants are required to conduct trials of immediate serial recall (ISR). Some lists are repeatedly presented (e.g., every third trial) as analogous to children 
Positional repetition in serial learning

being repeatedly exposed to certain sets of sound sequences (i.e., word forms). Performance on repeated lists improves with repetition compared to non-repeated lists. Therefore, it is assumed that repeated retention/recall of a sequence leads to knowledge of the sequence.

Analogous to the Hebb learning of a sequence, retention of subsequence level representations in STM is also expected to lead to acquisition of long-term knowledge of the representations. At least two forms of knowledge can be acquired at a subsequence level. One is knowledge of bi-element association and the other is knowledge of position-element association (Nakayama, Tanida, \& Saito, 2015).

A piece of evidence for acquired knowledge of bi-element frequency comes from phonotactic frequency effects on nonword recall. In a given language, a set of two elements (e.g., two phonemes or two morae) can frequently or infrequently occur as a subsequence of a larger sequence (e.g., a word form). Such occurrence frequency of bi-element subsequences predicted accuracy of ISR of nonwords containing the bi-element units by native speakers of the language (e.g., Gathercole et al., 1999; Nakayama et al., 2015; Tanida et al., 2015). The results are consistent with the assumption that native speakers of a language form a long-term association of the two elements via repeated experience of the association. More direct evidence comes from laboratory learning paradigms in which to-be-learned association frequency of two elements is experimentally manipulated. Majerus et al. (2004) had participants hear a stream of speech where (remote) transition probability of phonemes was manipulated. The results showed that subsequent nonword ISR accuracy was influenced by the transition probability. Botvinick (2005; Botvinick \& Bylsma, 2005) further demonstrated higher-order transition probability can be learned via and affect ISR. They showed that participants' performance including error patterns reflected experimentally manipulated artificial phonotactics where a class of items, rather than a 
Positional repetition in serial learning

specific item, more frequently follow the other class of items but items from the same class less frequently follow each other.

The acquisition of knowledge of position-element association, however, has been less investigated compared to the two types of knowledge above (i.e., sequence knowledge and bielement association knowledge). This study aimed to demonstrate that position-element association could be acquired as a form of long-term knowledge dissociated from other types of knowledge. Position-element association is a widely demonstrated and implemented serial ordering mechanism for STM (Botvinick \& Plaut, 2006; Brown, Preece, \& Hulme, 2000; Burgess \& Hitch, 2006; Farrell, 2012; Fischer-Baum \& McCloskey, 2015; Hartley \& Houghton, 1996; Henson, 1998, 1999; Hurlstone et al., 2014; O’Reilly \& Soto, 2001). Although details of implementation vary among models, the common core functioning is as follows. Each element in a sequence is associated with a corresponding positional representation, and the positional representation serves as a retrieval cue for ordered recall of the elements. For example, to remember a list “ABC," A, B, and C are associated with positions 1, 2, and 3, respectively. Ordered activation of positional representations (position 1, position 2, and position 3 ) leads to ordered activation of the elements to achieve ordered recall.

It is predicted that long-term position-element association is formed through repeated occupation by a specific element (e.g., "A") of a specific position (e.g., "position 1") and that it supports STM for the element occupying the position, even in a novel sequence. Consistent with this prediction, Nakayama, Tanida, \& Saito (2015) demonstrated the effects of position-element frequency in ISR of Japanese nonwords. Japanese vocabulary has many multi-moraic/multisyllabic words, and there is some stochastic regularity in the associations between mora and positions in a word, which are assumed to be learned by Japanese speakers. For example, a mora 
Positional repetition in serial learning

"ka" appeared 2,931,456 times as the first mora in four-mora words (e.g., "ka-ra-o-ke") and 433,327 times as the fourth mora in four-mora words (e.g., "a-me-ri-ka") in a Japanese word frequency database (Amano \& Kondo, 2000). This position-mora frequency explained the variance of recall accuracy of morae in nonwords (e.g., "ka" is better recalled as the first mora). Similarly, Dell and colleagues (Dell, Reed, Adams, \& Meyer, 2000; Warker \& Dell, 2006) provided evidence for long-term position-element association in the context of speech production. In the experiments, they trained participants to learn novel positional constraints. Participants were asked to read aloud nearly 400 sequences consisting of four consonant-vowelconsonant nonwords. Critically, these nonwords were constrained in such a manner that a certain consonant (e.g., "f') always appeared at a specific position (e.g., coda). Observed speech errors followed the newly learned positional constraints (e.g., "f" is a coda even though it is an error) indicating formation of long-term position-element associations. Therefore, the presence of longterm position-element associations is empirically supported.

The long-term position-element association seems to be dissociated from the whole sequence knowledge that is acquired through Hebb learning. Although the whole sequence repetition in the Hebb paradigm includes positional repetition of each item (otherwise whole lists could not be repeated), positional repetition itself does not strongly contribute to the standard Hebb effect. For example, positional repetition without whole repetition did not show an equivalent effect to whole list repetition even when scoring only the repeated items, at least with a relatively small number of repetitions that was enough for the occurrence of the Hebb repetition effect. In other words, the long-term position-element association effects were not observed in a situation where the Hebb effect was observed. Using a variant of the Hebb paradigm, Hitch, Fastame, and Flude (2005) tested the effects of positional repetition by 
Positional repetition in serial learning 6

presenting lists where only half of the elements were repeated in the same position (e.g., odd positions). Similarly, Cumming, Page, and Norris (2003) examined the performance of transfer lists where only half of the elements were presented in the same position of the Hebb list (i.e., the elements in either the odd or even positions were repeated in the transfer list). These studies demonstrated much larger effects of whole-list repetition compared to very limited effects of positional repetition suggesting the dissociation of sequence knowledge from position-element association knowledge (see also Majerus et al., 2012; Schwartz \& Bryden, 1971).

Position-element frequency knowledge is theoretically distinguished from bi-element frequency knowledge, even though bi-element frequency knowledge can be conceptualized as knowledge about frequency of relative position of elements or classes of elements (e.g., "B frequently follows A" and "A is frequently followed by B"). The long-term position representation we investigate here is representation of position relative to edges (i.e., start, and end in some models) of the sequence. Many models of short-term serial order memory utilize position code relative to edges of the sequence rather than relative to another element in the sequence (e.g., Henson, 1998; Henson \& Burgess, 1997). Our current question is whether such positional code exists as a form of long-term knowledge distinguished from knowledge of bielement or relative positions of items. Empirical evidence, indeed, suggests that position-element effects are statistically distinguishable from bi-element frequency effects (in this case, an element was a Japanese phonological unit - mora; Nakayama et al., 2015).

To summarize, previous research indicates presence of position-element association knowledge that is dissociable from other forms of knowledge. These studies, however, have limitations. Nakayama et al. (2015) demonstrated the position-mora frequency effects utilizing phonological structure of Japanese but they did not manipulate the to-be-learned structure per se. 
Positional repetition in serial learning

On one hand, utilizing natural language material/structure has an advantage of better ecological validity such that it allows investigating a consequence of very long-term (e.g., life-long) learning of language. On the other hand, observed effects might reflect confounding effects of unknown linguistic structures despite the great care for controlling for psycholinguistic properties of the materials. Sometimes, it would even be impossible to control all of the relevant psycholinguistic variables perfectly for materials from natural languages (Majerus et al., 2004). A laboratory learning paradigm can compensate this limitation as the to-be-learned structure can be experimentally controlled. Hitch et al. (2005) used manipulation and tested the positional repetition frequency in the laboratory overcoming the limitation of examining phonotactic frequency effects in a natural language (see also Cummings et al, 2003). Though the results provided evidence for dissociation of the Hebb repetition effect from position-element frequency effect, they did not show the positional frequency effect itself. Therefore, evidence for separable position-element association knowledge still needs to be accumulated.

The present study utilized a laboratory learning paradigm to demonstrate the acquisition of position-element association knowledge in a rigorously controlled setting. In a set of experiments participants were required to engage in a series of ISR. By manipulating the positional repetition frequency of items over the course of experiment, we tested whether positional repetition of an item leads to increase in recall accuracy of the item in the position. By experimentally controlling the other aspects of the to-be-learned structure, the position-element frequency effects would be safely assumed to reflect acquisition of the position-element association knowledge. We also compared the position-element frequency effects with the Hebb effect to directly show the dissociation. In contrast to previous research that demonstrated the dissociation by showing absence of positional frequency effect in the presence of the Hebb effect (Cummings 
Positional repetition in serial learning

et al., 2003; Hitch et al., 2005), we attempted to demonstrate successful acquisition of both types of knowledge with different rates of learning. Positional frequency effects were observed with natural language materials that were assumed to be overlearned by participants (Nakayama et al., 2015) whereas several repetitions were not sufficient to detect the positional frequency effects in the laboratory (Cummings et al., 2003; Hitch et al., 2005). Based on these findings we predicted that positional frequency effect would emerge only after extensive learning. The Hebb effect, in contrast, was predicted to emerge after a small number of repetitions as in previous research.

\section{Experiment 1}

First, an exploratory experiment was conducted. We knew that up to eight repetitions was not sufficient to detect the positional repetition effect (Cumming et al., 2003; Hitch et al., 2005) and that experiencing nearly 200 sequences was sufficient to detect systematic speech errors (Warker \& Dell, 2006); therefore, we began with as long an experiment as possible. In Experiment 1, participants underwent 704 ISR trials in three-day sessions. Throughout the experiment, some lists were repeatedly presented, and, importantly, each item was presented more often in specific positions than other positions. Based on the results of Experiment 1, we then manipulated the position-item frequency and list frequency in subsequent experiments. Using position-wise regressions to analyze the data, we examined a) whether position-item frequency showed position-selectivity (e.g., position-item frequency in position 2 typically influenced the recall accuracy for items in position 2) and b) whether position-item frequency effects emerge more gradually than list frequency effects. 
Positional repetition in serial learning

\section{Methods}

Participants. Six undergraduate students (three females) from Kyoto University participated in exchange for 6000 yen. Their age ranged from 19 to 24 years $(M=20.5)$. All participants were native Japanese speakers. Data from two additional participants were not included in the analysis because some data were missing due to a programming error. Excluding those data did not change the critical pattern of the results. Collecting a number of participants was costly given the length of the experiment; therefore, similar to previous studies, we collected a relatively small number of participants (e.g., Botvinick, 2005; Dell et al., 2000).

Materials. Two-hundred sixty-four six-item lists were created from a pool of ten two-mora Japanese nonwords (i.e., "to-nu," "he-ka," "re-chi," "so-mi," "na-te," "ru-ke," "su-ro," "shi-fu," "ta-ho," "ni-ma"). We used nonword items and avoided over-learned items to minimize the effect of learning history outside the laboratory. Participants could have learned associations among the positions and over-learned items such as digits, and that knowledge could have obscured the present learning effect (Majerus et al., 2012). Each nonword in our stimulus set had its own bi-mora frequency (e.g., the occurrence frequency of "te" after "na" for the nonword "nate" in a Japanese corpus); therefore, this involved some learning history. However, its occurrence frequency as a single unitary item is very low (almost zero) in the Japanese language; therefore, position-item association learning outside the laboratory is very unlikely.

Of the 264 lists, 44 were high-frequency learning lists that were repeated three times each day (i.e., nine repetitions in total), 44 were low-frequency learning lists that were repeated once each day (i.e., three repetitions in total), and 176 were transfer lists that were presented once throughout the experiment. Half of them (i.e., 88 lists) were presented at the end of the second day, and the other half were presented at the end of the third day. Sound files for each of the ten 
Positional repetition in serial learning

10

nonwords were created from sound files for individual mora. The mora sound files were recorded by a female who had absolute pitch, and the pitch was controlled to D3. The nonword sound files had a duration of $750 \mathrm{~ms}$. 
Positional repetition in serial learning

11

Table 1. Artificial phonotactics in Experiment 1

A: Planned positional frequency

\begin{tabular}{lcccccc} 
Item & Position 1 & Position 2 & Position 3 & Position 4 & Position 5 & Position 6 \\
\hline Item 0 & - & $\mathrm{H}$ & $\mathrm{M}$ & $\mathrm{M}$ & $\mathrm{M}$ & $\mathrm{L}$ \\
Item 1 & - & $\mathrm{L}$ & $\mathrm{H}$ & $\mathrm{M}$ & $\mathrm{M}$ & $\mathrm{M}$ \\
Item 2 & - & $\mathrm{M}$ & $\mathrm{L}$ & $\mathrm{H}$ & $\mathrm{M}$ & $\mathrm{M}$ \\
Item 3 & - & $\mathrm{M}$ & $\mathrm{L}$ & $\mathrm{M}$ & $\mathrm{H}$ & $\mathrm{M}$ \\
Item 4 & - & $\mathrm{L}$ & $\mathrm{M}$ & $\mathrm{M}$ & $\mathrm{M}$ & $\mathrm{H}$ \\
Item 5 & - & $\mathrm{H}$ & $\mathrm{M}$ & $\mathrm{L}$ & $\mathrm{M}$ & $\mathrm{M}$ \\
Item 6 & - & $\mathrm{M}$ & $\mathrm{H}$ & $\mathrm{M}$ & $\mathrm{M}$ & $\mathrm{L}$ \\
Item 7 & - & $\mathrm{M}$ & $\mathrm{M}$ & $\mathrm{H}$ & $\mathrm{L}$ & $\mathrm{M}$ \\
Item 8 & - & $\mathrm{M}$ & $\mathrm{M}$ & $\mathrm{L}$ & $\mathrm{H}$ & $\mathrm{M}$ \\
Item 9 & - & $\mathrm{M}$ & $\mathrm{M}$ & $\mathrm{M}$ & $\mathrm{L}$ & $\mathrm{H}$ \\
\hline
\end{tabular}

B: Actual positional frequency per 176 trials

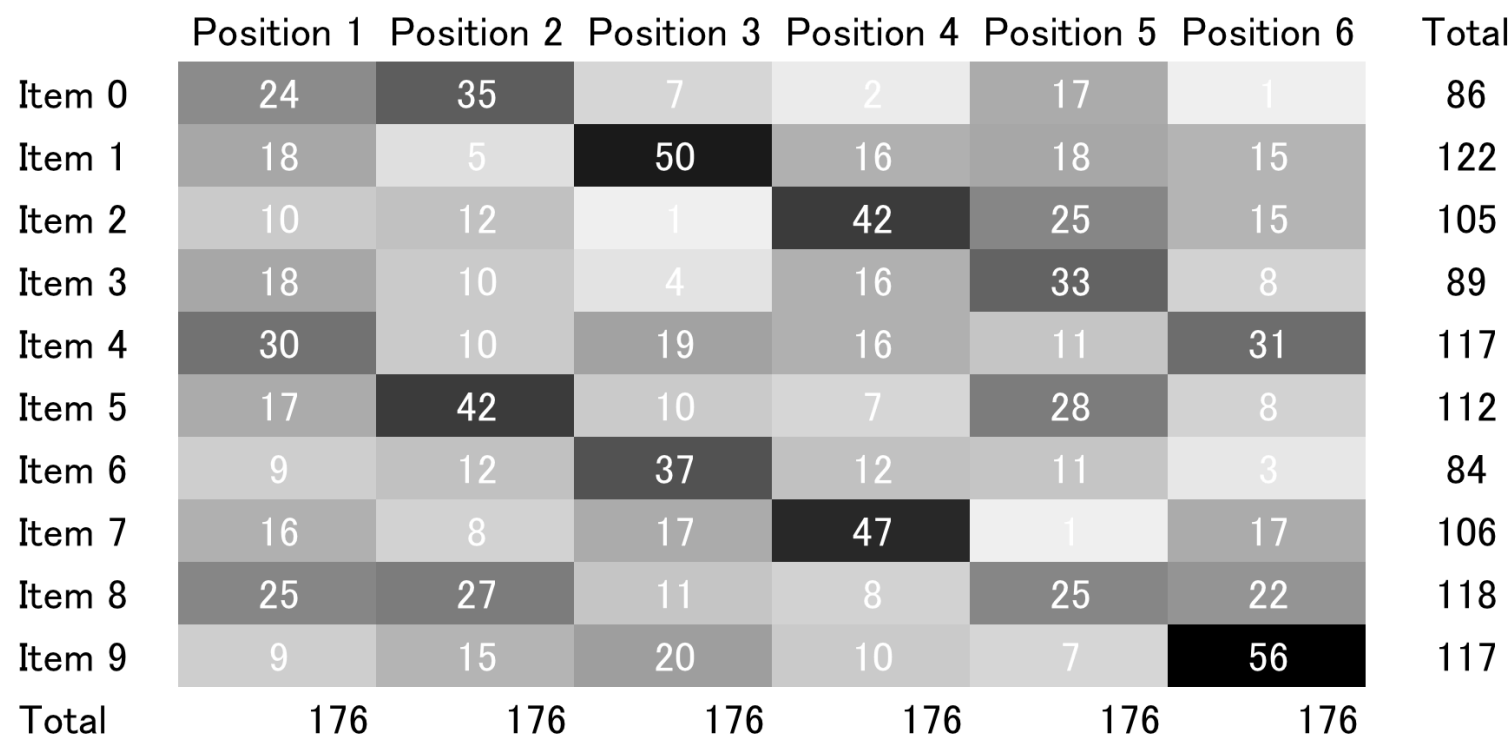

Note: Planned positional frequency is described in Table 1A. "H," "M," and "L" indicate high, middle, and low positional frequency, respectively. Table 1B indicates actual positional frequency of the learning lists that were selected based on Table 1A. Transfer lists are not 
Positional repetition in serial learning

12

included in this frequency calculation. Darker cells have higher frequency. Details of the selection procedure are described in the main text. 
Positional repetition in serial learning

13

Artificial phonotactics. To examine the position-item frequency effects, learning lists were constructed following artificial phonotactics. A planned positional frequency table was created (Table 1A). The table indicates which item tends to occupy which position. "H," "M," and "L" denote high, middle, and low positional frequency, respectively. For example, the second position would be occupied by Item 0 with high frequency and by Item 1 with low frequency. We unconstrained the positional frequency in Position 1 to make it easier to balance the frequency distribution in the other positions. The remaining five positions were constrained so that each position could be occupied as exactly two high-, six middle-, and two low-frequency item types. Similarly, each item can be allocated to exactly one high-, three middle-, and one low-frequency positions. Another reason for the exclusion of the first position from the position frequency manipulation is that the potential ceiling effect at that position might have made the positional frequency effect undetectable.

In addition to this positional frequency constraint, all lists were constrained not to share the first four items with the other lists. Since the Hebb list repetition effect transfers to those lists that share the first several items with the original list (e.g., Hitch et al., 2005), minimizing the effect of such onset matching is critical for showing a pure effect of positional repetition.

To implement the planned phonotactics, all possible permutations of six-item lists were generated. One list was randomly selected from each pool of lists where all the lists shared the first four items (i.e., neighborhood lists) to ensure that each selected list had no onset-shared neighborhood. All these no-neighborhood candidates were assigned selection probability based on planned positional frequency. If a list contained a high frequency position-item combination in one position (e.g., Item 0 in position 2), then the list had 2.7 times higher selection probability than those lists containing a middle frequency position-item combination in the position (e.g., 
Positional repetition in serial learning

14

Item 2 in position 2). Similarly, if a list contained a middle frequency position-item combination in one position (e.g., Item 2 in position 2), then that list had a 2.7 times higher selection probability than those lists containing a low frequency position-item combination in the position (e.g., Item 1 in position 2). The selection probability was accumulated through position 2 to 6 (i.e., product of the probabilities in these positions) to calculate the net selection probability for each list. Eighty-eight lists were selected according to the probability ratio. Through this procedure, we expected high-position frequency items to be presented 2.7 times more frequently than middle-position frequency items in each position. Similarly, middle-position frequency items were expected to be presented 2.7 times more frequently than low-position frequency items in each position. Of the lists, one-half (i.e., 44 lists) were randomly assigned as high-frequency lists and the other half (44 lists) as low-frequency lists. Table 1B indicates position-item frequency for the selected learning lists when high-frequency lists were presented three times and low-frequency lists once. The actual positional frequency of the learning list roughly reflects the planned positional frequency (Table 1A). For example, the "position 2-item 2" frequency was 2.4 times larger than the "position 2-item 1" frequency, and it was 1/2.92 times smaller than the "position 2-item 0" frequency. In total, each item appeared more frequently in one position than the other positions. In contrast to position-item frequency, total frequency was balanced among the items. Since the list-repetition frequency difference (i.e., one vs. three) was far smaller than the positional-frequency difference (e.g., position 2 -item 2 frequency 12 vs. position 2-item 0 frequency 35; Table 1B) the list repetition did not strongly affect the positional frequencies. Therefore, the list frequency was almost orthogonal to the positional frequency, though we statistically separated these effects during our analysis (see below). 
Positional repetition in serial learning

15

Transfer lists were selected based on the position-item frequencies in Table 1B. From noneighborhood candidates, lists were selected when the position-item frequency in each one of the positions 2 to 6 was either less than 10 (relatively low position-item frequency) or greater than 20 (relatively high position-item frequency). This selection criterion was intuitively determined from the actual phonotactic frequency distribution (Table 1B). Two sets of 88 lists were further selected using an algorithm and software package for the stochastic optimization of stimuli; SOS (Armstrong, Watson, \& Plaut, 2012) with this constraint. Position-item frequencies in each position were orthogonalized. Position-item frequencies for positions 2-6 were uniformly distributed into high- and low-frequency bands. The selection algorithm essentially used an optimization procedure. Starting from a randomly selected set of stimuli, satisfactions of constraints (e.g., correlations among position-item frequencies) were calculated for the current set and a set where one (random) stimulus was altered from the current set. Based on satisfaction, the next set was stochastically selected from the two. Each set of lists was assigned to the second day or the third day. Different nonwords were assigned to each item for different participants (e.g., "na-te" was assigned to item 1 for one participant, but to item 2 for another).

Procedure. On the first day, participants signed a consent form, received instructions, and performed a key familiarization task and ISR task. On the second and third days, participants performed an ISR task.

In the key familiarization task, participants were familiarized with nonword-key mapping that was subsequently used for recall of the nonword sequence in the ISR task. In each trial, a fixation stimulus (i.e., “*”) was presented for $1000 \mathrm{~ms}$ at the center of the computer display (I-O DATA [Ishikawa, Japan] LCD-AD199GEW), followed by an auditory presentation of a single 
Positional repetition in serial learning

16

nonword. After the presentation of the nonword, the fixation turned into a response cue (i.e., “?”), and ten nonwords in hiragana form appeared at the bottom half of the screen in a constellation analogous to the corresponding keys (i.e., "Q," "W," "E," "R," "V," "U,, "I," “O," "P," or "N" on a QWERTY keyboard). The nonword-key mapping was fixed for each participant and randomized across participants. Participants were asked to press a key corresponding to the heard nonword. At the key press, the associated sound file was played regardless of whether the response was correct or not, and no explicit feedback was provided. If participants pressed no key for 10 seconds or a key other than the ten keys, a beep sound was presented. The screen went blank after the presentation of the nonword or beep. The next trial began $1000 \mathrm{~ms}$ after the blank screen. Participants were instructed to prioritize accuracy over speed and completed 300 trials. In each trial, the presented nonword was randomly selected from the ten nonwords. All visual stimuli in this task and the ISR task were presented in white MS Mincho font against a black background. All auditory stimuli in this task and the ISR task were presented via headphones (SONY [Tokyo, Japan] MDR-CD900ST), and the experiment was conducted in a soundattenuated room.

In the ISR task, participants began each trial by pressing the space bar. A fixation stimulus (i.e., “*”) was presented at the center of a computer display for $1000 \mathrm{~ms}$, followed by the auditory presentation of six consecutive nonwords with a 250-ms pause following each nonword. After the last pause, a recall cue (i.e., "?") and the ten nonwords appeared on the screen, as they did in the key familiarization task. Participants were asked to recall the six nonwords in the presented order by pressing the corresponding keys, and they were encouraged to recall or guess all the nonwords. At each key press, the associated sound file was played regardless of whether the response was correct or not, and no explicit feedback was provided. If participants pressed no 
Positional repetition in serial learning

17

key for 10 seconds or a key other than the ten keys, a beep sound was presented. Participants heard the entire nonword or beep as the key press was ignored until the end of the sound file. The screen became blank after the presentation of the sixth sound file. "Hot Soup Processer" (http://hsp.tv/) controlled the experiment, running on an EPSON (Nagano, Japan) Endeavor MR4000. A randomizing software, "Mix" (van Casteren \& Davis, 2006), pseudo-randomized the trial order. The algorithm avoided repetition of the same item in the same position in two consecutive trials. In previous research, positional intrusion from the (immediate) previous list had been observed and been assumed to reflect short-term rather than long-term position-element association (Cumming et al., 2003; Henson, 1999). Therefore, immediate positional repetition was avoided to ensure that the observed position-item frequency effects were not the products of short-term position-item association (i.e., positional intrusions from the previous list that were fortunately correct in the current trial).

Participants rested for three minutes after every 88 trials. The learning lists were presented on each day, and the transfer lists were presented in the last 88 trials of the second or third day.

The experimental protocol in this and subsequent experiments was approved by the research ethics committee for psychological experiments at the Graduate School of Education, Kyoto University.

\section{Data analysis}

In all, $0.3 \%$ of the trials were excluded from the accuracy analysis because of response omissions. Responses were coded as omissions if participants pressed no key for 10 seconds or a key other than the ten keys. Trials with omissions were excluded from the accuracy analysis; however, they were included in the frequency calculation reported below. Using mixed-effects 
Positional repetition in serial learning

18

logistic regressions (Jaeger, 2008) in the statistical software package R (R Core Team, 2013), we conducted a position-wise analysis. We conducted six independent regression analyses where the item recall accuracy for each list position was the dependent variable. Only the items recalled at the correct position were regarded as correct. Random effects included intercepts for participants and lists, as well as slopes of participants for position-item frequency in the position (e.g., P1F for position 1 regression; see below). Lists were regarded as the same based on the artificial phonotactics (e.g., "026587”) rather than assigned nonwords or keys. Note that the assignment of an item/list to a nonword or key was randomized across participants, and the same lists in terms of nonwords or key could not be experienced by different participants. Fixed effects included control variables and predictor variables.

Control variables included trial number and day ${ }^{1}$. Trial number of each tested trial was included to control for a general practice effect. The number was not reset at the beginning of each day (e.g., the first trial in the second day was coded as the 177th trial as there were 176 trials in the first day). Though we tried to use a raw trial number, the model estimations failed to converge. The inspection of the learning curve as a function of the trial number suggested that a non-linear practice effect existed throughout the three days (Fig. 1A; see also Melton, 1963); therefore, we used a base 10 logarithm of the raw trial number. Day (with centered coding of -1, 0, or 1) was also included controlling any additional effects between days (e.g., consolidations during sleep).

\footnotetext{
$1 \quad$ Nakayama et al. (2015) included accuracy in other positions as a control variable in order to examine the relatively pure effect of position-element frequency; however, this study did not include these variables for the sake of fair comparison. As those authors discussed, correlations in accuracy among positions are likely generated by whole list-level representations. This study aimed to estimate and compare list frequency effects with position-element frequency effects, and controlling for accuracy in other positions was not appropriate for this purpose.
} 
Positional repetition in serial learning

Predictor variables included position-item frequencies and list frequency. Therefore, the effect of each type of frequency was mutually controlled when examining one type of effect. Similar to linear regressions, logistic regressions allow us to estimate increase in (log) odds (i.e., probability of correct recall relative to that of incorrect recall) per one-unit increase in each predictor variable under the condition of the other variables being equal. Therefore, regression coefficients (i.e., odds ratios) are estimations of the learning (i.e., the increase in accuracy) per one-unit increase of frequency variables.

Position-item frequency for each position was calculated in a trial-by-trial manner (i.e. cumulative frequency). For example, when item 1 was presented at position 2 in the first, third, and eighth trials, the position-item frequency for position 2, or P2F, for each trial was 1,2 , and 3, respectively. This frequency was counted independently for each position. In other words, if the item appeared in the other positions (e.g., position 4), it was not included in the frequency counts for position 2. Though we tried to use raw frequency, the model estimations failed to converge. The inspection of the learning curve suggested a non-linear position-item effect throughout the three days (i.e., stable positional frequency effect after 176th trial; Fig. 1A); therefore, we used a base 10 logarithms of the raw frequency as predictor variables. There were six position-item frequencies (P1F to P6F, with the number indicating position). Effects of these position-item frequencies (i.e., coefficients for each positional frequency) would reflect long-term positionelement associations as (1) position-free item frequencies were controlled to balance out, (2) general practice effect was partialed out by statistically controlling for the effect of the trial number of each tested trial, and (3) list frequency effect was statistically controlled as well as almost orthogonalized at the list construction. The list frequency was calculated in a trial-by-trial 
Positional repetition in serial learning $\quad 20$

manner. It varied from 1 to 9 for high-frequency learning lists and 1 to 3 for low-frequency learning lists. All transfer lists had a frequency value of 1 .

\section{Results and Discussion}

Regression coefficients (odds ratios) of predictors are reported in Figures 1B and 2B (Detailed statistics are reported in Table A1).

Position-item frequency. For demonstrative purposes, Figure 1A presents the recall accuracy as a function of positional frequency and trial number. To make the figure intuitively understandable, we used the nominal frequency category in the planned positional frequency table (Table 1A). Those position-item combinations that are planned to be "high frequency" were categorized as "high frequency" irrespective of actual frequency. Those position-item combinations that were planned to be "low frequency" or "middle frequency" were categorized as "low and middle frequency" irrespective of actual frequency. Across second to sixth positions, mean accuracy was calculated for each frequency category and trial number category (e.g., 1-88, 89-176). As can be seen, position-item frequency emerged on the first day (i.e., by 176th trial) and became stable on the subsequent days including the transfer lists. A formal regression analysis confirmed this pattern (Figure 1B, Table A1). Position-item frequencies exhibited position selectivity affecting mostly and significantly $(z \mathrm{~S}>2.09$; Table A1) the accuracy in corresponding positions (e.g., P6F influenced accuracy in position 6). Though the positional frequency distribution was not explicitly manipulated at position 1 , the recall accuracy showed sensitivity to the (incidental) trial-by-trial fluctuation of positional frequency. 
Positional repetition in serial learning $\quad 21$

List frequency. For demonstrative purposes, Figure 2A presents the recall accuracy averaged across positions, participants, and lists as a function of day/list frequency and list type. Recall accuracy for learning lists increased with repetition. However, the maximum accuracy for learning lists did not exceed the accuracy of the transfer lists (i.e., lists that were only experienced once, sometimes called filler lists) in each day. Consistent with this visual inspection, formal regression analyses for each position revealed a non-significant or even a negative effect for list repetition (Figure 2B, Table A1). The lack of list frequency effect may not be surprising as it was shown that the list repetition effect disappears when the items for repeated and intervening lists are drawn from a closed set, at least when the repeating lists were separated by more than five trials (Melton, 1963; Page, Cumming, Norris, McNeil, \& Hitch, 2013). Notably, this experiment used a closed set (i.e., items were drawn from a ten-item set for sixitem lists). Repeated lists were also separated by very many trials, even on the first day ( $M=$ $43.7, S D=36.6$ trials). Therefore, the lack of list frequency effect might be attributable to this specific experimental setting. In summary, Experiment 1 demonstrated gradually emerging position-item frequency effects, in contrast to absent or even negative whole-list frequency effects. 
Positional repetition in serial learning $\quad 22$

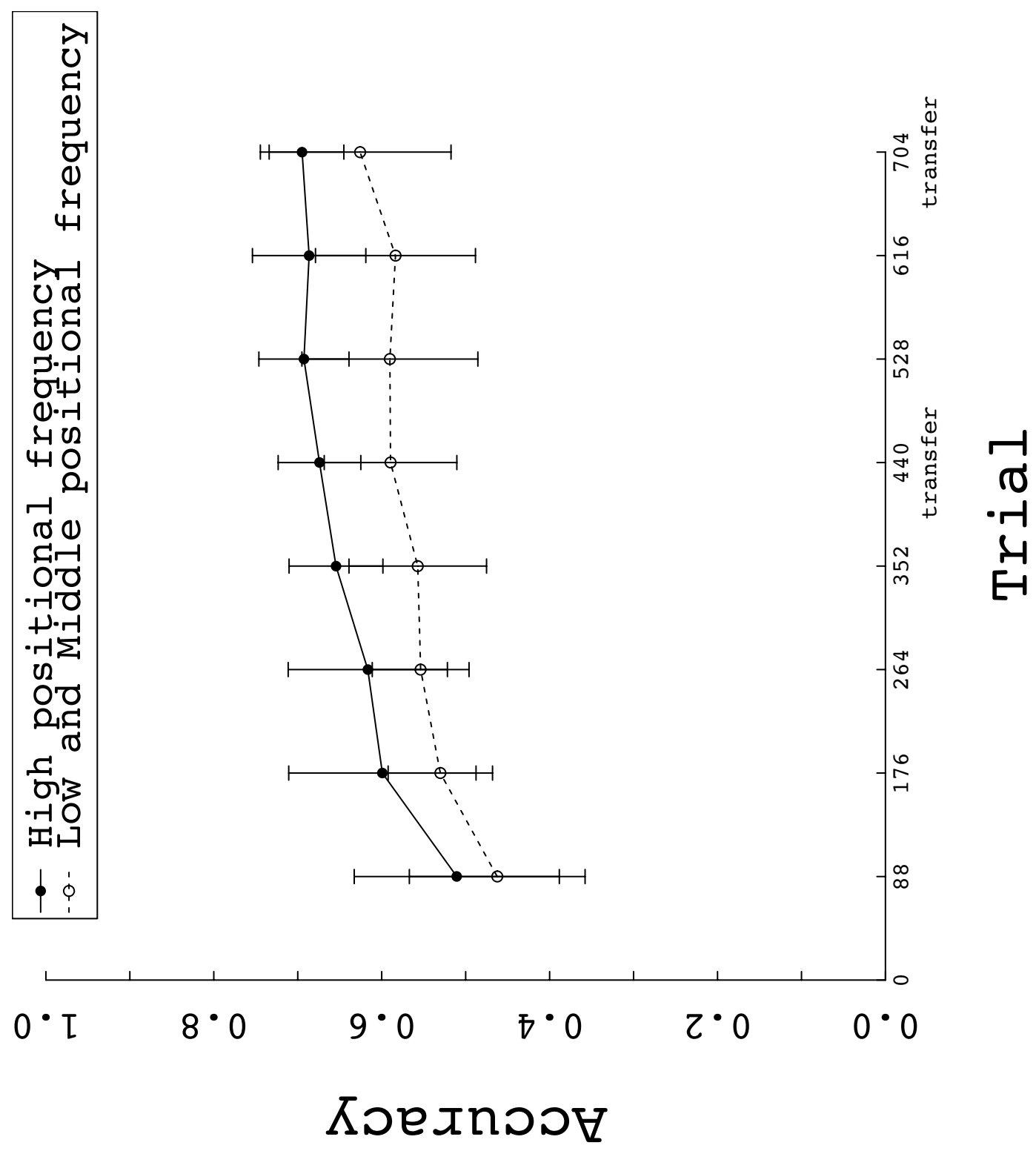

A 
Positional repetition in serial learning 23

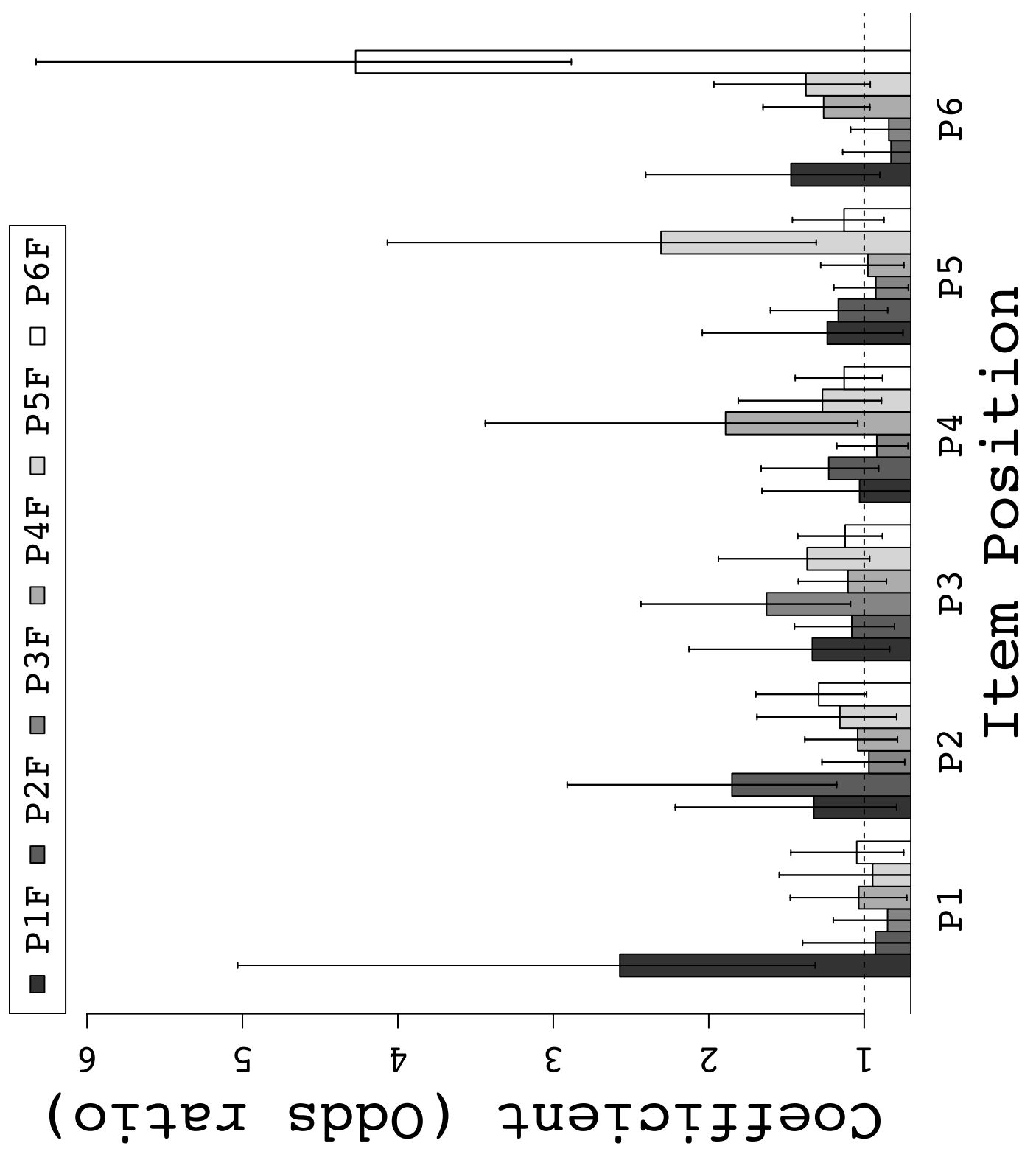


Positional repetition in serial learning

24

Figure 1. Position-item frequency effects in Experiment 1 
Positional repetition in serial learning $\quad 25$

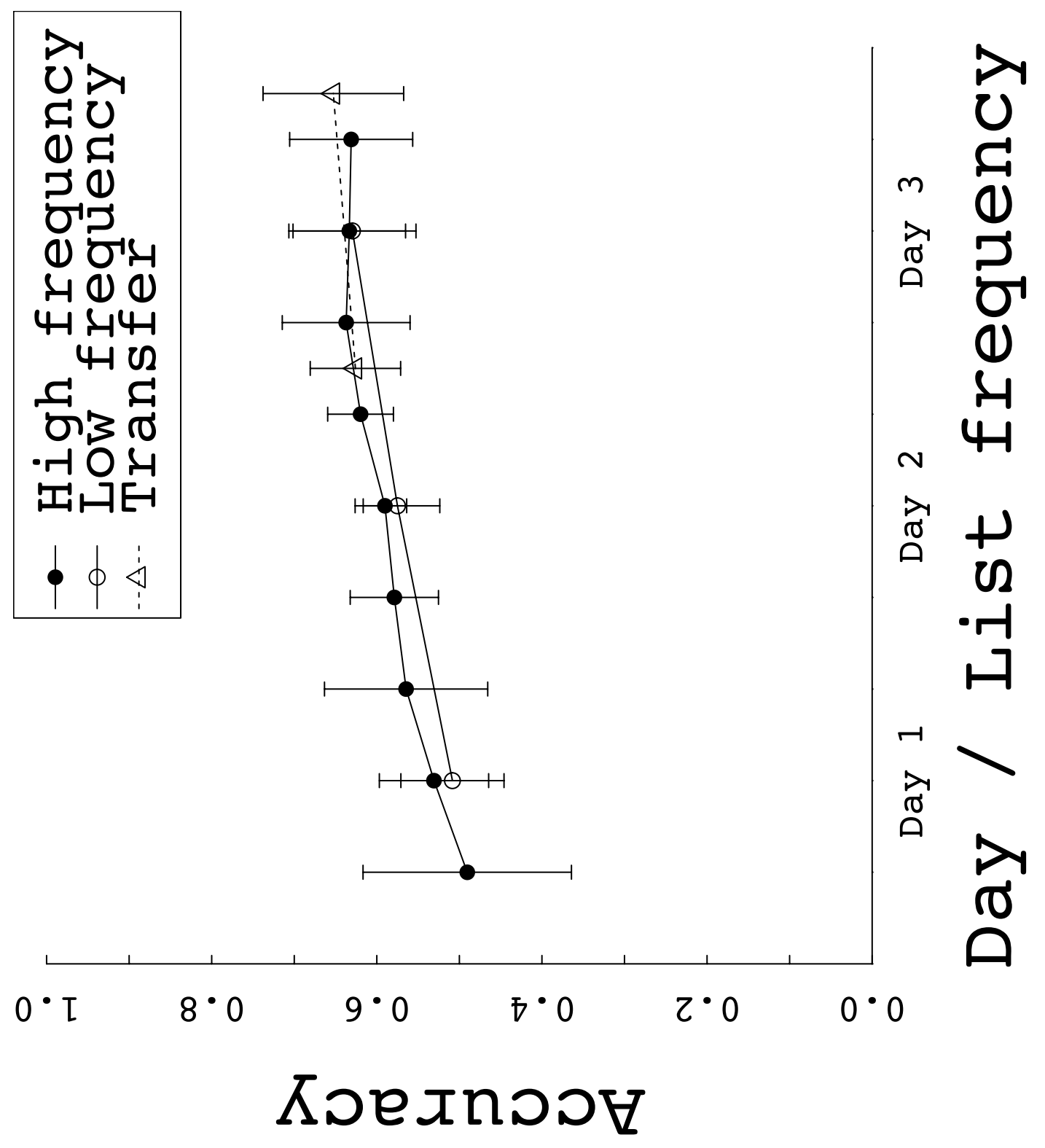


Positional repetition in serial learning 26

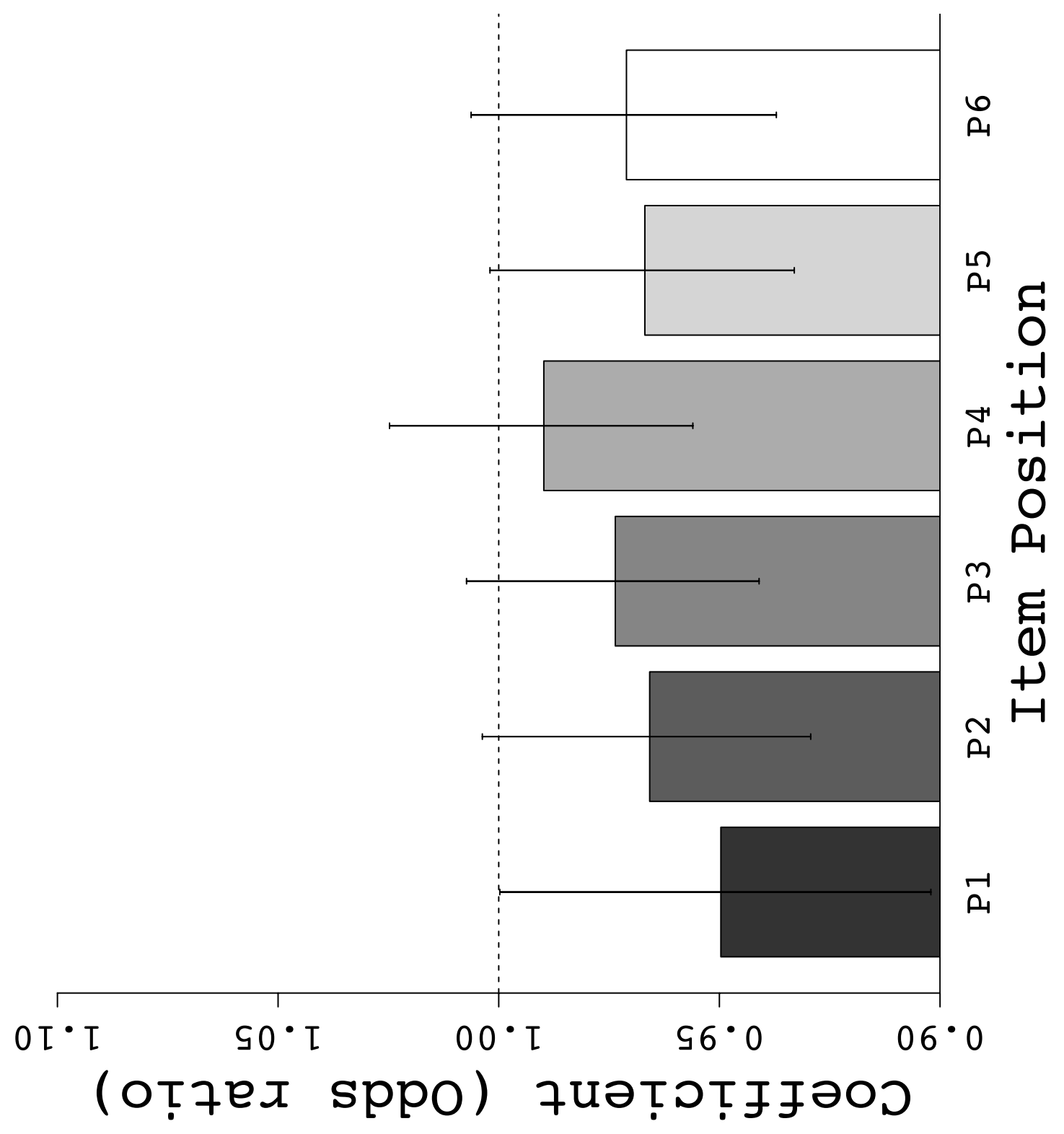


Positional repetition in serial learning

27

Figure 2. List frequency effects in Experiment 1 


\section{Experiment 2}

Although Experiment 1 provided a piece of evidence for gradually learned long-term position-element association, the experiment did not show the classic Hebb repetition (i.e., list frequency) effect. The lack of a list repetition effect prevented us from directly comparing the learning rate for position-item and list frequency. Therefore, in the subsequent experiments we attempted to replicate the present position-item frequency effect and the classic list frequency effect. Two separate, but matched experiments manipulated the two frequencies, and the learning rates were compared ${ }^{2}$. Experiment 2 manipulated the position-item frequency. Experiment 3 manipulated the list frequency. The inspection of Figure 1A indicated that the positional frequency effect emerged as early as the first day though the effect was not statistically detectable probably because of the small sample size of participants. In subsequent experiments, the length of the experiments was reduced to one day and the number of participants was increased to roughly match the number of data points (i.e., the number of participants multiplied by the number of trials) of Experiment 1. Therefore, Experiment 2 aimed to establish the positional frequency effects in a one-day experiment to compare the effects with the Hebb repetition effect in Experiment 3.

2 We tried to manipulate the two frequencies within one experiment; however, it turned out that keeping the number of intervening lists small between repeated lists strongly conflicted with avoiding immediate positional repetition and that the pseudo-randomizing algorithm by "Mix" could not find a solution with a reasonable number of attempts. In Experiment 4, simultaneous manipulation of the positional and list frequency was achieved by making the Hebb list comprise only high positional repetition frequency items rather than completely crossing the two types of frequency. 
Positional repetition in serial learning

29

\section{Methods}

Participants. Twenty-nine undergraduate students (sixteen female) from Kyoto University participated in exchange for 1500 yen. Their age ranged from 19 to 27 years $(M=21.3)$. All participants were native Japanese speakers. Data from an additional one participant were not included in the analysis because part of the data was missing due to a programming error.

Materials. All aspects of the materials were identical to the previous experiment except that 164 six-item lists were created and presented only once for each participant.

Artificial phonotactics. New phonotactics were created and used for list construction. Table $2 \mathrm{~A}$ is the planned positional frequency table. The same procedure selected a set of lists as in the previous experiment, except for the following modification. The "middle" frequency was not used. Selection probability of a "high" position-item combination was four times larger than that of a "low" position-item combination. Using this set of frequency distributions and selection probabilities, a "high" frequency position-item combination is expected to be repeated, on average, once in four trials (i.e., separated by three trials). This expectation value is within the range of the effective repetition separation of the Hebb repetition (Melton, 1963; Page et al., 2013). The resultant phonotactics are indicated in Table 2B. 
Positional repetition in serial learning $\quad 30$

Table 2. Artificial phonotactics in Experiment 2

A: Planned positional frequency

\begin{tabular}{rrrrccc}
\hline Item & Position 1 & Position 2 & Position 3 & Position 4 & Position 5 & Position 6 \\
\hline Item 0 & - & $\mathrm{H}$ & $\mathrm{L}$ & $\mathrm{L}$ & $\mathrm{L}$ & $\mathrm{L}$ \\
Item 1 & - & $\mathrm{H}$ & $\mathrm{L}$ & $\mathrm{L}$ & $\mathrm{L}$ & $\mathrm{L}$ \\
Item 2 & - & $\mathrm{L}$ & $\mathrm{H}$ & $\mathrm{L}$ & $\mathrm{L}$ & $\mathrm{L}$ \\
Item 3 & - & $\mathrm{L}$ & $\mathrm{H}$ & $\mathrm{L}$ & $\mathrm{L}$ & $\mathrm{L}$ \\
Item 4 & - & $\mathrm{L}$ & $\mathrm{L}$ & $\mathrm{H}$ & $\mathrm{L}$ & $\mathrm{L}$ \\
Item 5 & - & $\mathrm{L}$ & $\mathrm{L}$ & $\mathrm{H}$ & $\mathrm{L}$ & $\mathrm{L}$ \\
Item 6 & - & $\mathrm{L}$ & $\mathrm{L}$ & $\mathrm{L}$ & $\mathrm{H}$ & $\mathrm{L}$ \\
Item 7 & - & $\mathrm{L}$ & $\mathrm{L}$ & $\mathrm{L}$ & $\mathrm{H}$ & $\mathrm{L}$ \\
Item 8 & - & $\mathrm{L}$ & $\mathrm{L}$ & $\mathrm{L}$ & $\mathrm{L}$ & $\mathrm{H}$ \\
Item 9 & - & $\mathrm{L}$ & $\mathrm{L}$ & $\mathrm{L}$ & $\mathrm{L}$ & $\mathrm{H}$ \\
\hline
\end{tabular}

B: Actual positional frequency in the entire experiment (164 trials)

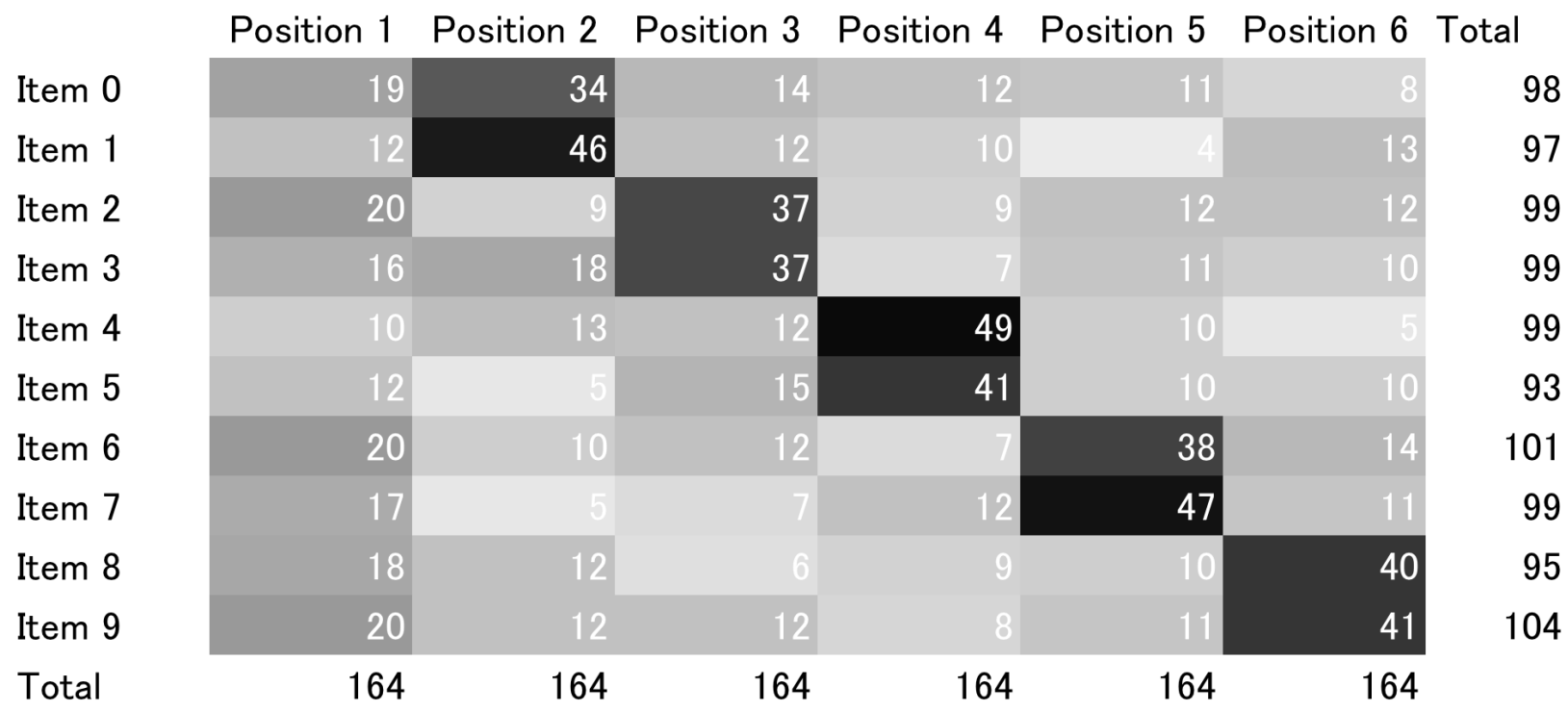

Note: Planned positional frequency is described in Table 2A. "H" and "L" indicate high and low positional frequency, respectively. Table 2B indicates actual positional frequency of the presented lists that were selected based on Table 2A. Details of the selection procedure are described in the main text. 
Positional repetition in serial learning

31

Procedure. Procedure was similar to the first day of Experiment 1. Participants experienced 300 trials of the key-familiarization task and 164 trials of the ISR task with a 3minute rest at the halfway point of the task. The other aspects that differed from Experiment 1 were that a Dell (Round Rock, TX) Latitude E4300 computer was used for running the experiment and participants answered a questionnaire ${ }^{3}$ at the end of the experiment.

\section{Data analysis}

Following the same criteria and procedure as in Experiment 1, 1.6\% of the trials were excluded from the accuracy analysis. As in Experiment 1, six independent mixed-effects logistic regressions were conducted. Random effects included intercepts for participants, lists, and slopes of participants for position-item frequency in the position.

The control variable, as a fixed effect, included a base 10 logarithm of the trial number.

Predictor variables included position-item frequencies. Frequencies were calculated in a trial-by-trial manner, and raw frequency values were used for the purpose of direct comparison with the (raw) list frequency effect in Experiment 3. As in Experiment 1, coefficients for frequency variables indicate how much the probability of correct recall (relative to probability of incorrect recall) increases per increase in the frequency (e.g., how much the accuracy of position 2 item increases if the position-item combination repeated once more). Learning rates for

3 We have not reported the results of the questionnaire, as they do not contribute to our main purpose. However, for the sake of open science, we are reporting the contents of the questionnaire. It included demographic variables and open-ended questions about the experiment, awareness of the positional or list repetition (for Experiments 2 and 3, respectively, and both for Experiment 4), and the strategy used. The strategy items included rehearsal, remembering the first sound (letter) of each nonword, remembering the spatial location of each nonword in the recall phase, remembering the location of the key for each nonword, and grouping patterns (including non-grouping). 
Positional repetition in serial learning

32

position-item frequency and list frequency were compared by comparing the coefficients for these frequency variables across the two experiments.

\section{Results and Discussion}

Regression coefficients of position-item frequencies are reported in Figure 3B. The coefficients are reported in terms of odds ratios with $95 \%$ confidence intervals in the figure for the purpose of comparison with the list frequency effect in Experiment 3. Note that the range of the vertical axis in Figure 3B is scaled to match that in Figure 4B. Detailed statistics are reported in Table A2.

For demonstrative purposes, Figure 3A shows the recall accuracy as a function of positional frequency and trial number. To make the figure intuitively comprehensible, we used the nominal frequency category in the planned positional frequency table (Table 2A). Those position-item combinations that were planned to be "high frequency" were categorized as "high frequency" irrespective of actual frequency. Those position-item combinations that were planned to be "low frequency" were categorized as "low frequency" irrespective of actual frequency. Across positions 2 through 6, mean accuracy was calculated for each frequency category and trial number category (e.g., 1-41, 42-84). Experiment 2 replicated the results of Experiment 1. Position-item frequency effect gradually increased up to the 84th trials and stabilized after that. Formal regression analysis confirmed this pattern (Figure 3B) where coefficients for positional frequencies roughly corresponds to difference in slopes of the two frequency categories in Figure 3A. Position-item frequencies exhibited position selectivity affecting mostly and significantly the accuracy of corresponding positions ( $z \mathrm{~s}>2.27$; Table A2). For example, P6F influenced 
Positional repetition in serial learning

33

accuracy in position 6. Notably, in contrast to the larger P6F effect than the other positions in Experiment 1, the size of the P6F effect was similar to the other positions in this experiment. The recency effect may not be a robust effect although it is difficult to directly compare the results of the two experiments because of some minor differences between the two experiments. As in Experiment 1, the recall accuracy at position 1 showed sensitivity to the (incidental) trial-by-trial fluctuation of positional frequency despite the fact that the positional frequency distribution was not explicitly manipulated at position 1 . Therefore, we successfully replicated the position-item frequency observed in Experiment 1. Regression coefficients (odds ratios) showed gradual learning ranging from 1.01 to 1.09 . These coefficients are compared with those of the list frequency in Experiment 3. 
Positional repetition in serial learning

34
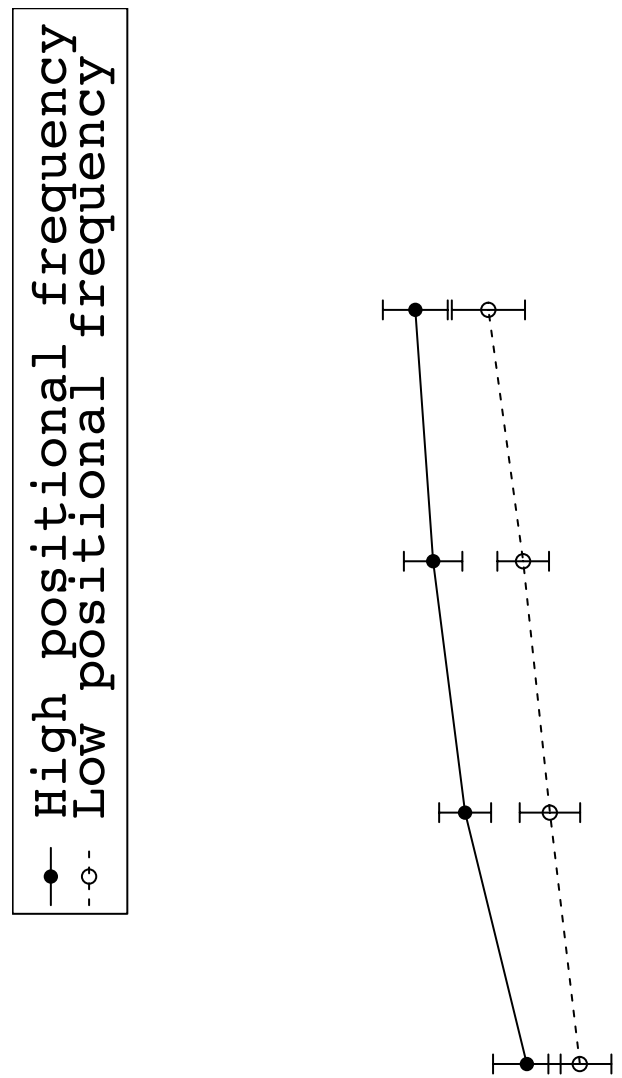

ֻே

$\stackrel{m}{\sim}$

$\sqrt{10}$

$\sigma$

$\cdot-1$

$\underset{\infty}{\infty}$

E-1

$\stackrel{-}{\sim}$

o

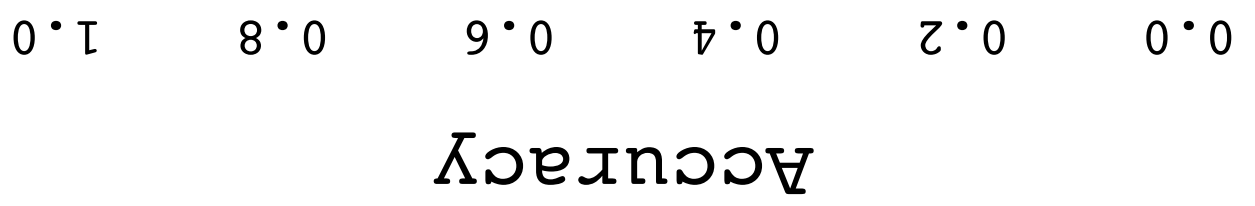

A 
Positional repetition in serial learning

35
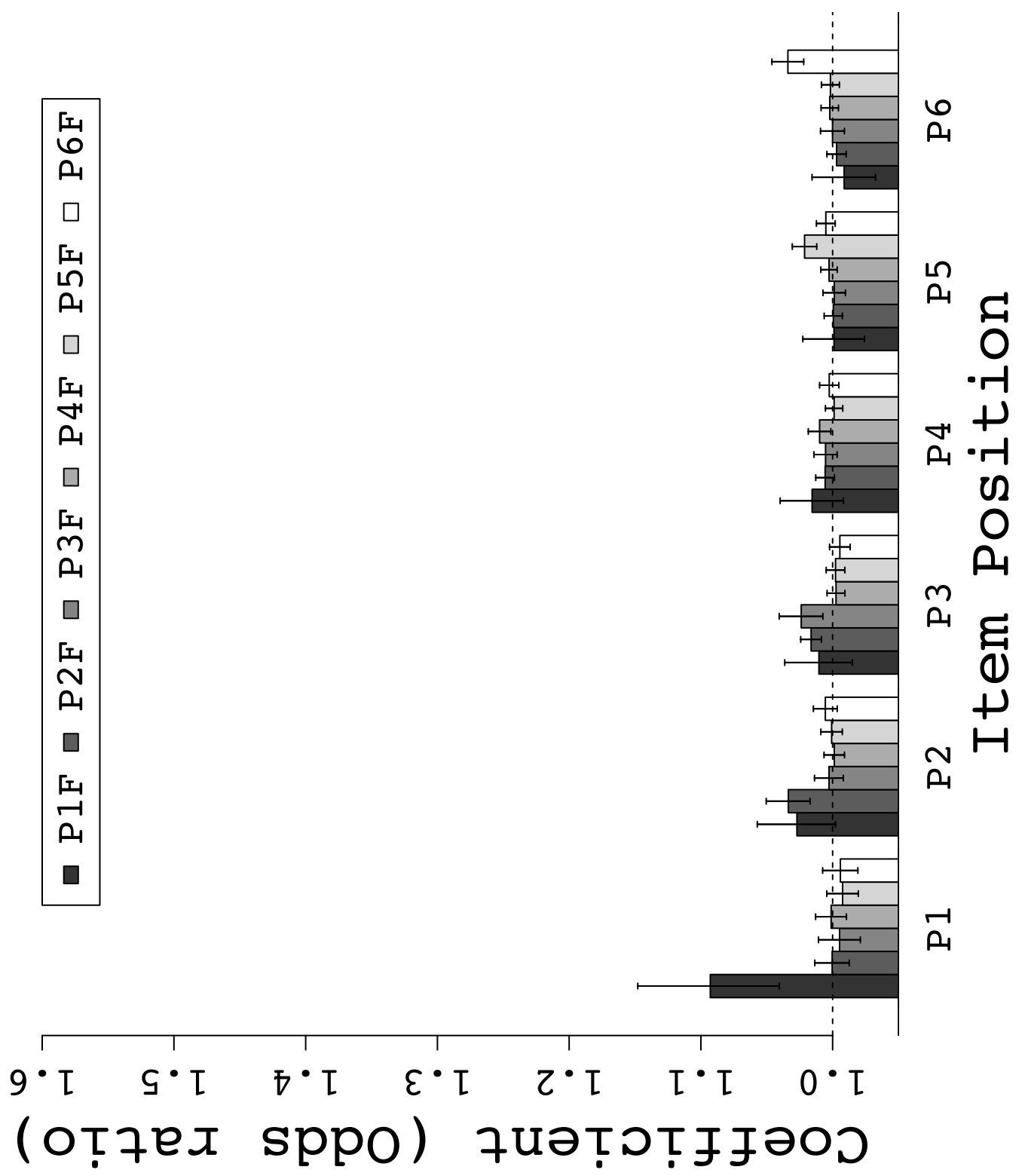
Positional repetition in serial learning $\quad 36$

Figure 3. Position-item frequency effects in Experiment 2 
Positional repetition in serial learning

37

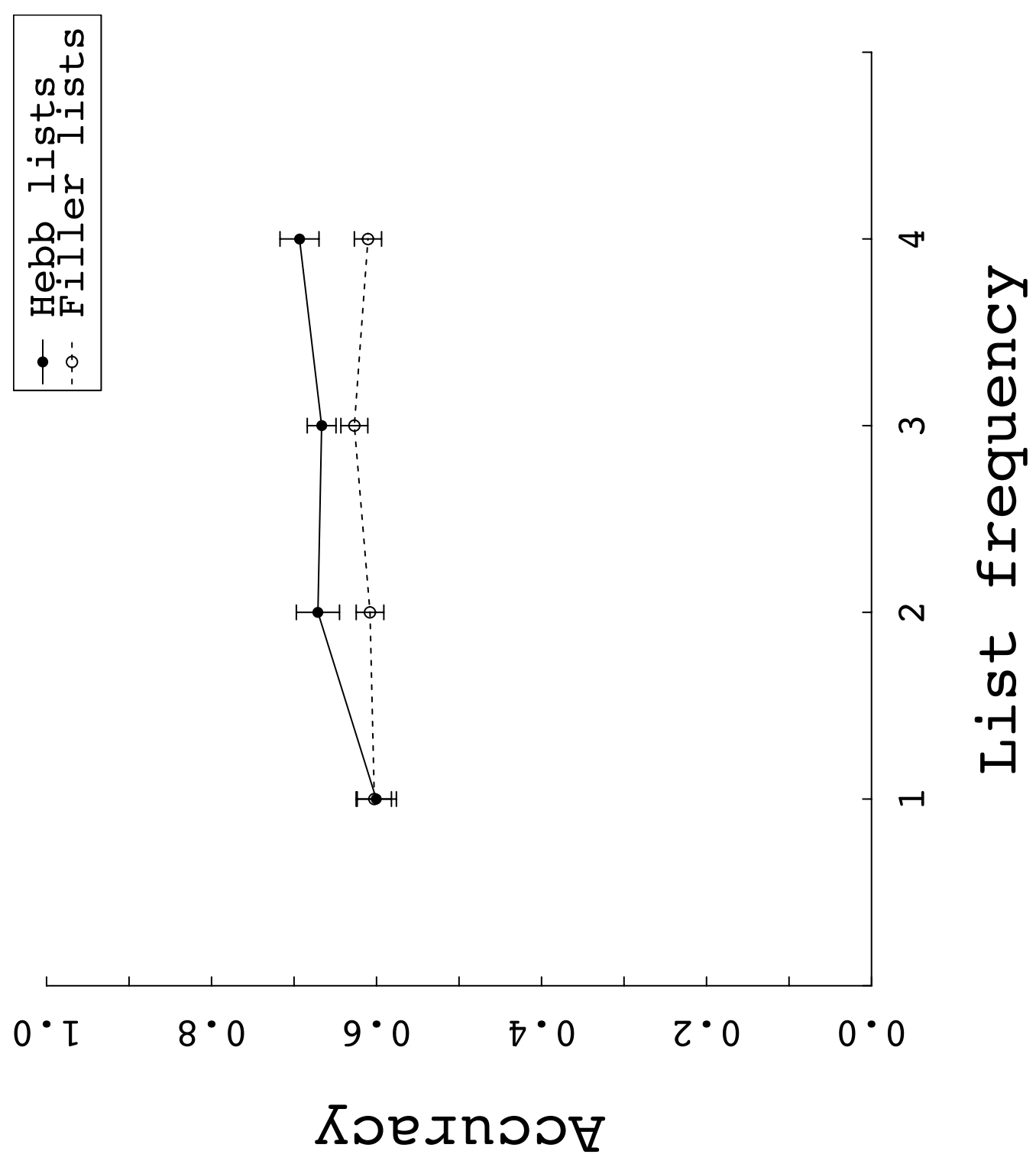

A 
Positional repetition in serial learning 
Positional repetition in serial learning $\quad 39$

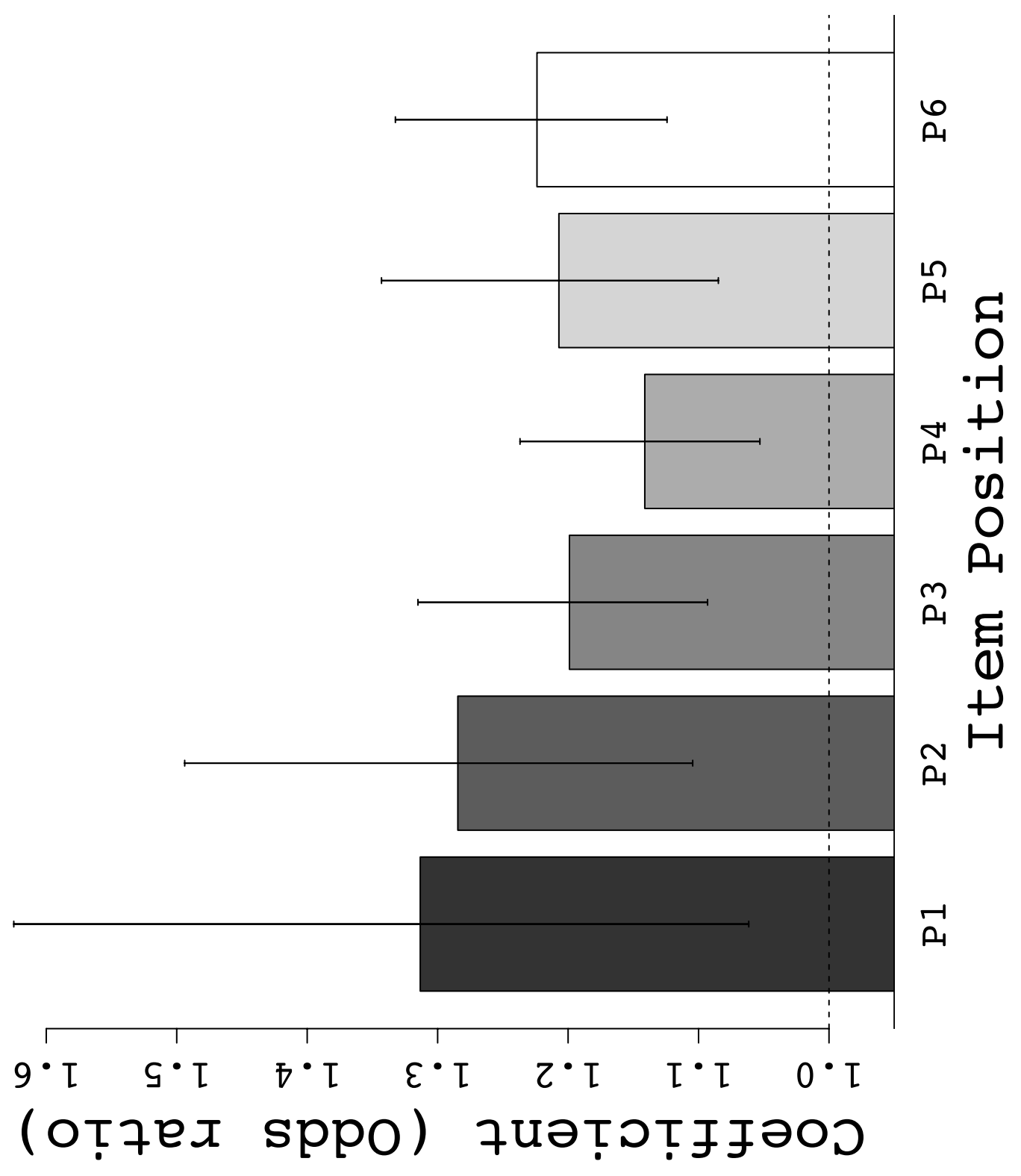


Positional repetition in serial learning $\quad 40$

Figure 4. List frequency effects in Experiment 3

\section{Experiment 3}

Experiment 3 aimed to establish the Hebb list repetition effects in a one-day experiment to compare the effects with positional repetition effects in Experiment 2. As described above, a critical factor that prevents replication of the list frequency effect may be the number of intervening lists between repeated lists. Therefore, the number of intervening lists was set to two, which was within the effective range of the Hebb repetition (e.g., Hebb, 1961).

\section{Methods}

Participants. Twenty-eight undergraduate students (18 males) from Kyoto University participated in exchange for 1500 yen. Their age ranged from 18 to 37 years $(M=20.7)$. All participants were native Japanese speakers. Data from two additional participants were not included in the analysis because one participant did not complete the experiment and the other participant had participated in Experiment 1.

Materials. All aspects of the materials were identical to the previous experiments except that 108 six-item lists were randomly selected from the "no-neighborhood candidates" and randomly assigned to Hebb or filler lists. Each of the 12 Hebb lists was presented four times with each presentation sandwiched by two of 96 filler lists. The list repetition locally causes fluctuation in positional frequency (i.e., the same items were repeated in the same position during the list repetition); however, globally, position-item frequencies were balanced in this set.

Procedure. The procedure was identical to that of Experiment 2 except for the number of trials in the ISR task (i.e., 144 trials). 
Positional repetition in serial learning

41

\section{Data analysis}

Following the same criteria and procedure as the previous experiments, $1.8 \%$ of trials were excluded from the accuracy analysis. Six independent mixed-effects logistic regressions were conducted. Random effects included intercepts for participants, lists, and slope of participants for list frequency.

The control variable included a base 10 logarithm of the trial number as a fixed effect.

Predictor variables included list frequency. Frequency was calculated in a trial-by-trial manner, and raw frequency values were used for the purpose of direct comparison with the position-item frequency effect in Experiment 2. The regression coefficients for list and positional frequency were compared.

\section{Results and Discussion}

Regression coefficients of list frequency are reported in Figure 4B. The coefficients are reported in terms of odds ratios with $95 \%$ confidence intervals in the figure for the purpose of comparison with the position-item frequency effect in Experiment 2. Detailed statistics are reported in Table A3.

For demonstrative purpose, Figure 4A shows the recall accuracy averaged across participants, individual lists, and positions 2 to 6 as a function of list frequency and list nature. As the Hebb list is presented in every third trial, the accuracy of the sandwiching non-repeated filler lists is plotted in the corresponding frequency condition. As can be seen, the accuracy increased even with this small number of repetitions. The formal regression analysis confirmed this pattern (Figure 4B) where the coefficient for list frequency corresponds to difference in 
Positional repetition in serial learning

42

slopes of the two list categories in Figure 4A. The list frequency effects were significant in all positions $(z \mathrm{~s}>2.51$; Table A3). The odds ratios ranged from 1.14 to 1.31 among positions.

In sum, Experiment 3 successfully replicated the classic Hebb repetition effect after a modification of the number of intervening lists between repeated lists. This result confirms that the lack of the Hebb effect in Experiment 1 is attributable to the long repetition lag. Under this assumption, the position-item frequency effects were a) observed using a laboratory learning paradigm and b) slowly learned compared to list frequency effects. The coefficients for positional frequency and list frequency (i.e., odds ratios per repetition) were 1.01-1.09 vs. 1.141.31 where $95 \%$ confidence intervals for the two frequency variables did not overlap in all positions except for position 1 (Figures 3 and 4; Tables A2 and A3).

\section{Experiment 4}

Although the comparison of the positional repetition effects in Experiment 2 and the Hebb repetition effects in Experiment 3 indicated faster learning in Hebb repetition, these experiments and comparisons have some limitations. Critically, the lags between the highly frequent positional repetitions in Experiment 2 were not fixed and the expected value (i.e., three intervening trials) was slightly larger than the (fixed) lags between the Hebb lists in Experiment 3 (i.e., two intervening trials). The longer lag might have prevented the learning by positional repetition and resulted in a spurious difference between the positional repetition and the Hebb repetition. Therefore, Experiment 4 directly compared the positional repetition and the Hebb repetition with the repetition lag being completely controlled. In addition, the nonword-key mapping was fixed for each participant in the previous experiments. It is possible that 
Positional repetition in serial learning

43

participants learned associations between temporal position and key/finger location rather than nonwords. Experiment 4 randomized the key mapping in each trial to confirm that the locus of positional repetition learning was in the phonological domain, rather than in spatial and/or motor ones. 
Positional repetition in serial learning

Table 3. Artificial phonotactics in Experiment 4

A: Planned positional frequency

\begin{tabular}{cccccc}
\hline Item & Position 1 & Position 2 & Position 3 & Position 4 & Position 5 \\
\hline Item 0 & $\mathrm{H}$ & $\mathrm{L}$ & $\mathrm{L}$ & $\mathrm{L}$ & $\mathrm{L}$ \\
Item 1 & $\mathrm{H}$ & $\mathrm{L}$ & $\mathrm{L}$ & $\mathrm{L}$ & $\mathrm{L}$ \\
Item 2 & $\mathrm{L}$ & $\mathrm{H}$ & $\mathrm{L}$ & $\mathrm{L}$ & $\mathrm{L}$ \\
Item 3 & $\mathrm{L}$ & $\mathrm{H}$ & $\mathrm{L}$ & $\mathrm{L}$ & $\mathrm{L}$ \\
Item 4 & $\mathrm{L}$ & $\mathrm{L}$ & $\mathrm{H}$ & $\mathrm{L}$ & $\mathrm{L}$ \\
Item 5 & $\mathrm{L}$ & $\mathrm{L}$ & $\mathrm{H}$ & $\mathrm{L}$ & $\mathrm{L}$ \\
Item 6 & $\mathrm{L}$ & $\mathrm{L}$ & $\mathrm{L}$ & $\mathrm{H}$ & $\mathrm{L}$ \\
Item 7 & $\mathrm{L}$ & $\mathrm{L}$ & $\mathrm{L}$ & $\mathrm{H}$ & $\mathrm{L}$ \\
Item 8 & $\mathrm{L}$ & $\mathrm{L}$ & $\mathrm{L}$ & $\mathrm{L}$ & $\mathrm{H}$ \\
Item 9 & $\mathrm{L}$ & $\mathrm{L}$ & $\mathrm{L}$ & $\mathrm{L}$ & $\mathrm{H}$ \\
\hline
\end{tabular}

B: Actual positional frequency per 16 trials

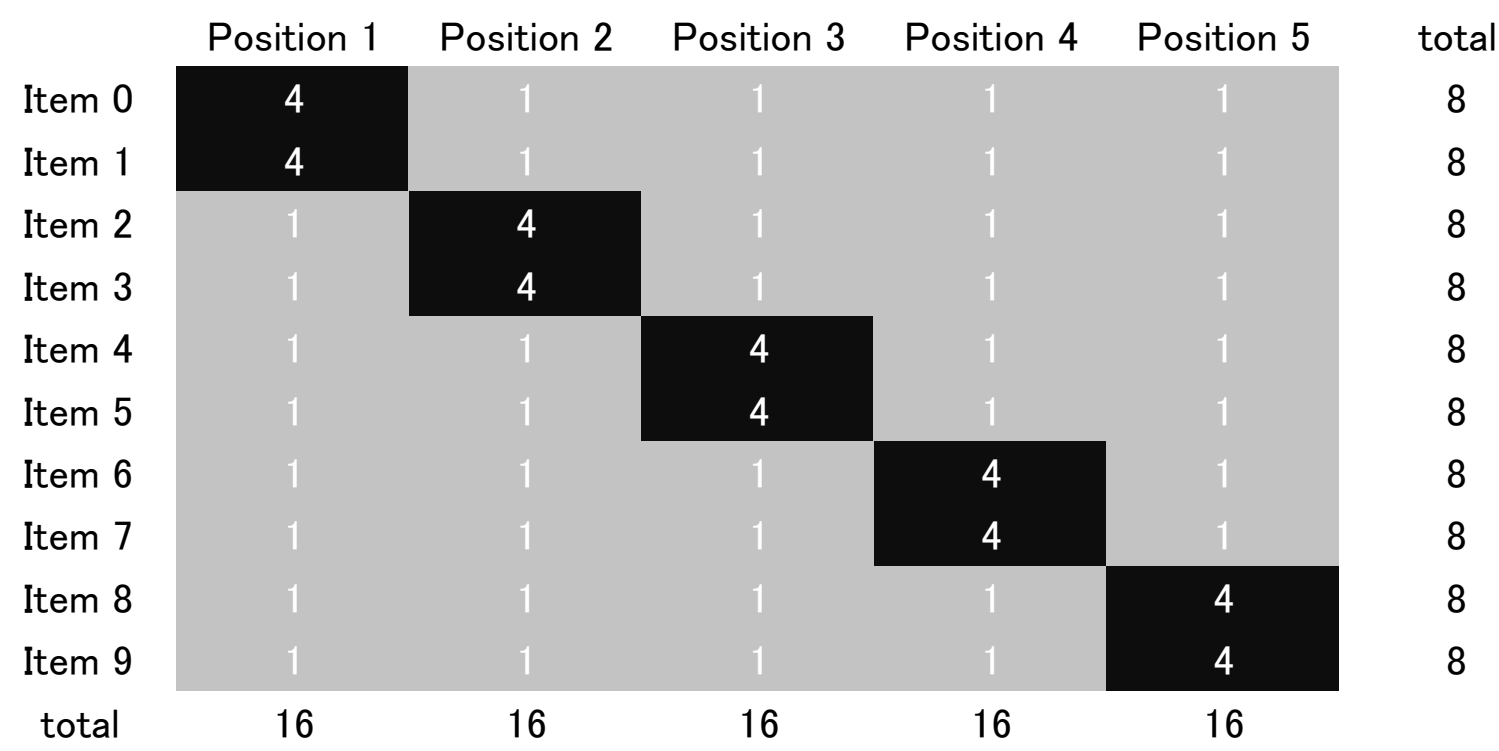


Positional repetition in serial learning

45

C: An example of list presentation

\begin{tabular}{|c|c|c|c|c|c|c|c|c|}
\hline Block & Sub-block & Trial & List type & Position 1 & Position 2 & Position 3 & Position 4 & Position 5 \\
\hline \multirow[t]{16}{*}{ Block 1} & Sub-block 1 & Trial 1 & Mixed-PF & 0 & 3 & 4 & 9 & 2 \\
\hline & & Trial 2 & Hebb & 1 & 2 & 5 & 6 & 8 \\
\hline & & Trial 3 & Mixed-PF & 5 & 4 & 8 & 7 & 9 \\
\hline & & Trial 4 & Filler & 4 & 6 & 7 & 8 & 0 \\
\hline & Sub-block 2 & Trial 5 & Mixed-PF & 0 & 3 & 4 & 1 & 5 \\
\hline & & Trial 6 & Hebb & 1 & 2 & 5 & 6 & 8 \\
\hline & & Trial 7 & Mixed-PF & 6 & 8 & 3 & 7 & 9 \\
\hline & & Trial 8 & Filler & 7 & 9 & 0 & 4 & 3 \\
\hline & Sub-block 3 & Trial 9 & Mixed-PF & 0 & 3 & 4 & 5 & 6 \\
\hline & & Trial 10 & Hebb & 1 & 2 & 5 & 6 & 8 \\
\hline & & Trial 11 & Mixed-PF & 3 & 0 & 1 & 7 & 9 \\
\hline & & Trial 12 & Filler & 9 & 7 & 2 & 0 & 4 \\
\hline & Sub-block 4 & Trial 13 & Mixed-PF & 0 & 3 & 4 & 2 & 1 \\
\hline & & Trial 14 & Hebb & 1 & 2 & 5 & 6 & 8 \\
\hline & & Trial 15 & Mixed-PF & 8 & 5 & 6 & 7 & 9 \\
\hline & & Trial 16 & Filler & 2 & 1 & 9 & 3 & 7 \\
\hline \multirow[t]{6}{*}{ Block 2} & Sub-block 5 & Trial 17 & Mixed-PF & 3 & 4 & 5 & 6 & 9 \\
\hline & & Trial 18 & Hebb & 0 & 2 & 4 & 7 & 8 \\
\hline & & Trial 19 & Mixed-PF & 1 & 3 & 6 & 5 & 2 \\
\hline & & Trial 20 & Filler & 6 & 9 & 3 & 2 & 1 \\
\hline & & $\cdot$ & & & & . & & \\
\hline & & $\cdot$ & & & & $\cdot$ & & \\
\hline \multirow[t]{4}{*}{ Block12 } & Sub-block 48 & Trial 189 & Mixed-PF & 3 & 2 & 4 & 9 & 8 \\
\hline & & Trial 190 & Hebb & 1 & 3 & 5 & 7 & 9 \\
\hline & & Trial 191 & Mixed-PF & 0 & 5 & 1 & 6 & 2 \\
\hline & & Trial 192 & Filler & 4 & 1 & 6 & 8 & 3 \\
\hline
\end{tabular}

Note: Planned positional frequency is described in Table 3A. "H" and " $\mathrm{L}$ " indicate high and low positional frequency, respectively. Table 3B indicates actual positional frequency of the presented lists for every 16 trials. Details of the selection procedure are described in the main text. Table $3 \mathrm{C}$ exemplifies a presentation order. PF: Positional frequency 
Positional repetition in serial learning $\quad 46$

\section{Methods}

Participants. Thirty undergraduate students from Kyoto University (18 males) participated in exchange for 1500 yen. Their age ranged from 18 to 24 years $(M=20.8)$. All participants were native Japanese speakers.

Materials and Presentation schedule. Planned and actual phonotactics are shown in Tables 3A and 3B. In Table 3A, high frequency position-item combinations are denoted " $\mathrm{H}$ " and low position-item combinations are denoted "L" with the former presented four times more frequently than the latter. The list repetition frequency was manipulated within each block and the position-item frequency was manipulated throughout the experiment. To directly compare the positional repetition and the Hebb repetition effect, three types of five-item lists were constructed. Hebb lists were the lists that were repeated four times with three intervening trials. The Hebb lists were controlled to consist of only "high" frequency position-item combinations. Mixed positional frequency lists were the lists that were not repeated at the list level; however, they consisted of high and low frequency position-item combinations. Low positional frequency lists or filler lists were not repeated at the list level and consisted of only low frequency positionitem combinations. As exemplified in Table 3C, these lists were presented in four trials of one sub-block. In each sub-block, one mixed positional frequency list, one Hebb list, one mixed positional frequency list, and one filler list were presented in this order. Within the sub-block, one of the two high frequency position-item combinations at each serial position (e.g., item 0 in position 1; shaded cell in Table 3C) was assigned to one of the two mixed frequency list (e.g., 03492). The other high frequency combination (e.g., item 1 in position 1) was assigned to the Hebb list (e.g., 12568). Each of the two mixed frequency lists had two or three high positional 
Positional repetition in serial learning

47

frequency combinations (e.g., 034XX and XXX79 with X denoting low-positional frequency items). These high frequency position-item combinations were repeated through consecutive four sub-blocks (i.e., one block; Table 3C). Low frequency position-item combinations were presented exactly once in one block with random assignment to each possible positional slot (i.e., non-shaded cells in Table 3C). This presentation procedure allowed us to completely equate 1) the number of intervening trials of whole list and positional repetition and 2) the accumulated positional frequency of the Hebb list and high frequency combinations in the mixed frequency lists throughout the experiment. Sixteen blocks (i.e., 48 sub-blocks or 192 trials) were selected from a set of possible blocks to satisfy the following constraints: a) no list except for the Hebb list was repeated, b) each Hebb list was presented no more than four times, and c) no item was repeated within a list ${ }^{4}$. In all, 12 Hebb lists, 96 mixed frequency lists, and 48 filler lists were selected for 192 trials. A full example of presentation is shown in Appendix A. The order of the blocks was randomized for each participant. As in the previous experiments, a set of nonwords were randomly assigned as item 0 to item 9 for each participants.

Procedure. The procedure was identical to that of Experiments 2 and 3 except for a) nonword-key mapping was randomized for each trial and b) the number of practice trials was reduced to 50 trials because participants were no longer able to learn a specific nonword-key mapping but each nonword itself. c) Each list consisted of five nonwords rather than six.

\section{Data analysis}

$4 \quad$ Though the lists may share some items from the onset (i.e., onset-neighborhoods), avoiding these neighborhood lists could have made the selection extremely difficult. As the effects were controlled in previous experiments, we gave up controlling for the effect in the current experiment in exchange for controlling other important aspects such as repetition lag. 
Positional repetition in serial learning

48

Following the same criteria and procedure as the previous experiments, some trials were excluded. Because of programming error, three trials of the first three participants failed to show precise key mapping on the display; therefore, these trials were also excluded. In all, $1.0 \%$ of trials were excluded from the accuracy analysis. Five independent mixed-effects logistic regressions were conducted. Random effects included intercepts for participants, lists, and slope of participants for positional frequency in the list and list frequency.

The control variable included the sub-block number as a fixed effect. Among four types of lists in one sub-block, positional frequency for high and low-positional frequency items were equated within each frequency category and it was straightforward to use the sub block number as a control variable for general practice effect.

Predictor variables included positional frequency and list frequency. Frequency was calculated in a trial-by-trial manner, and raw frequency values were used. As in Experiments 2 and 3, coefficients were compared to compare the learning rates for position-item frequency and list frequency. 
Positional repetition in serial learning

49

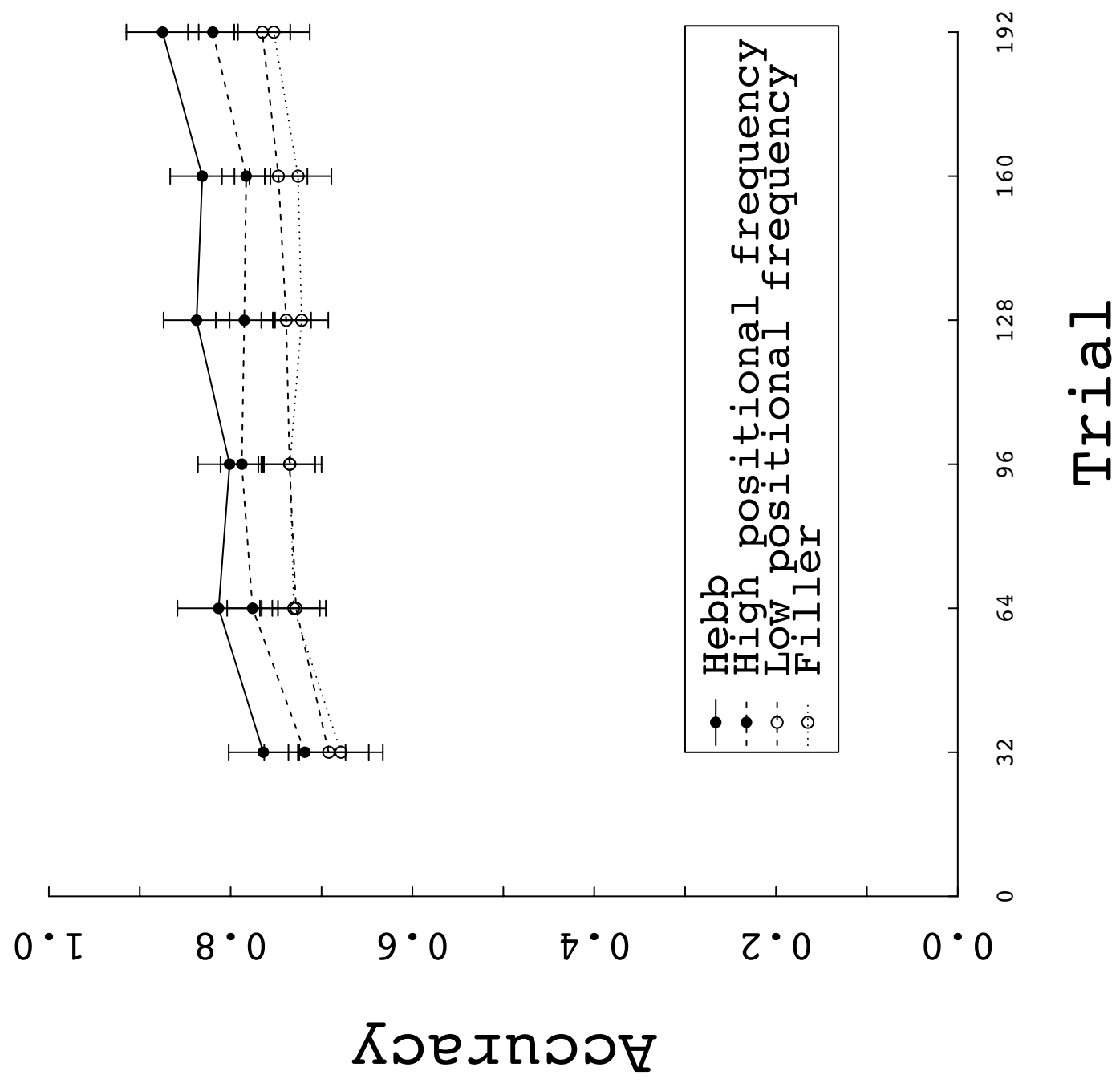

A 
Positional repetition in serial learning $\quad 50$

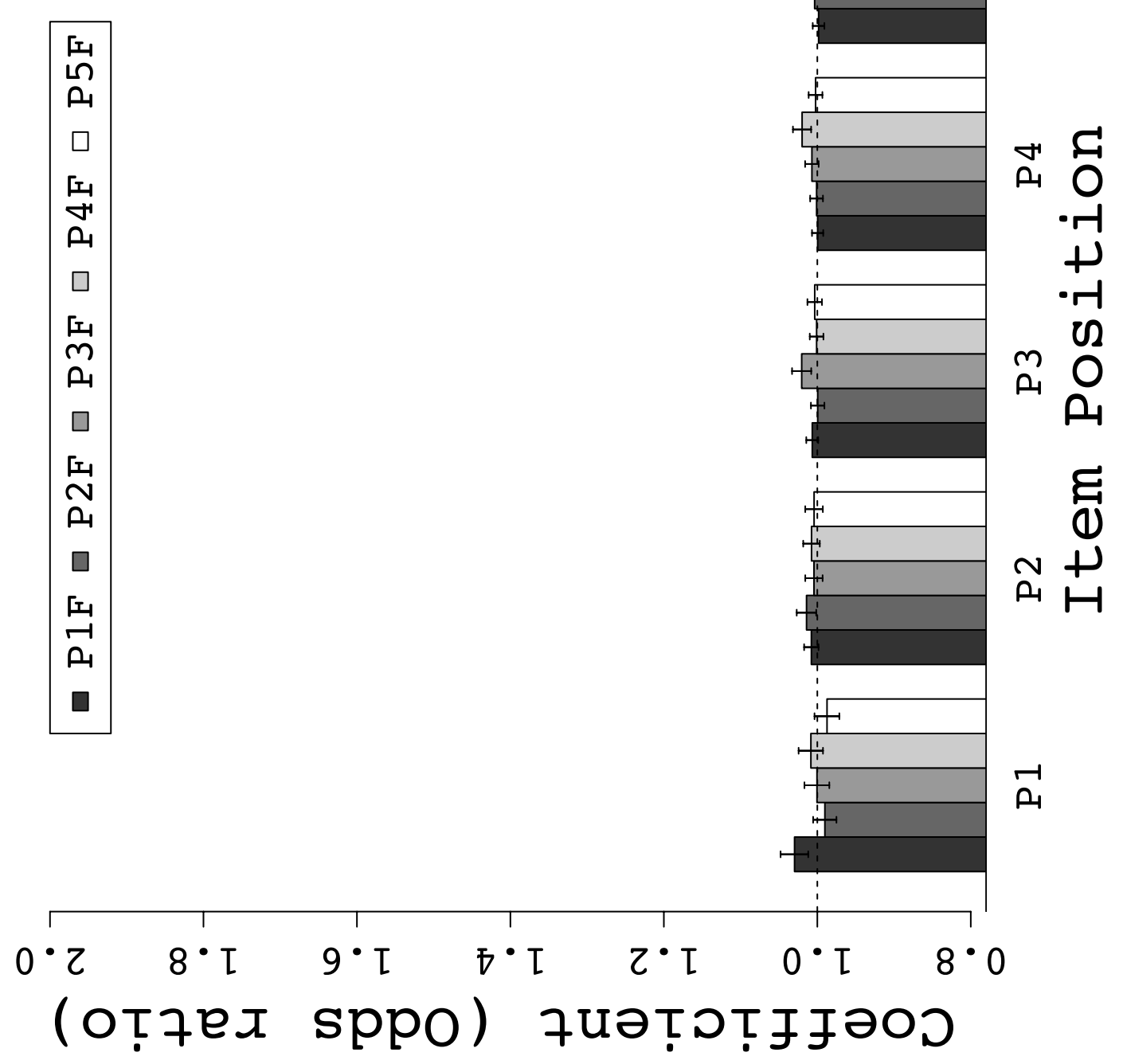

\section{B}

Figure 5. Position-item frequency effects in Experiment 4 
Positional repetition in serial learning

51
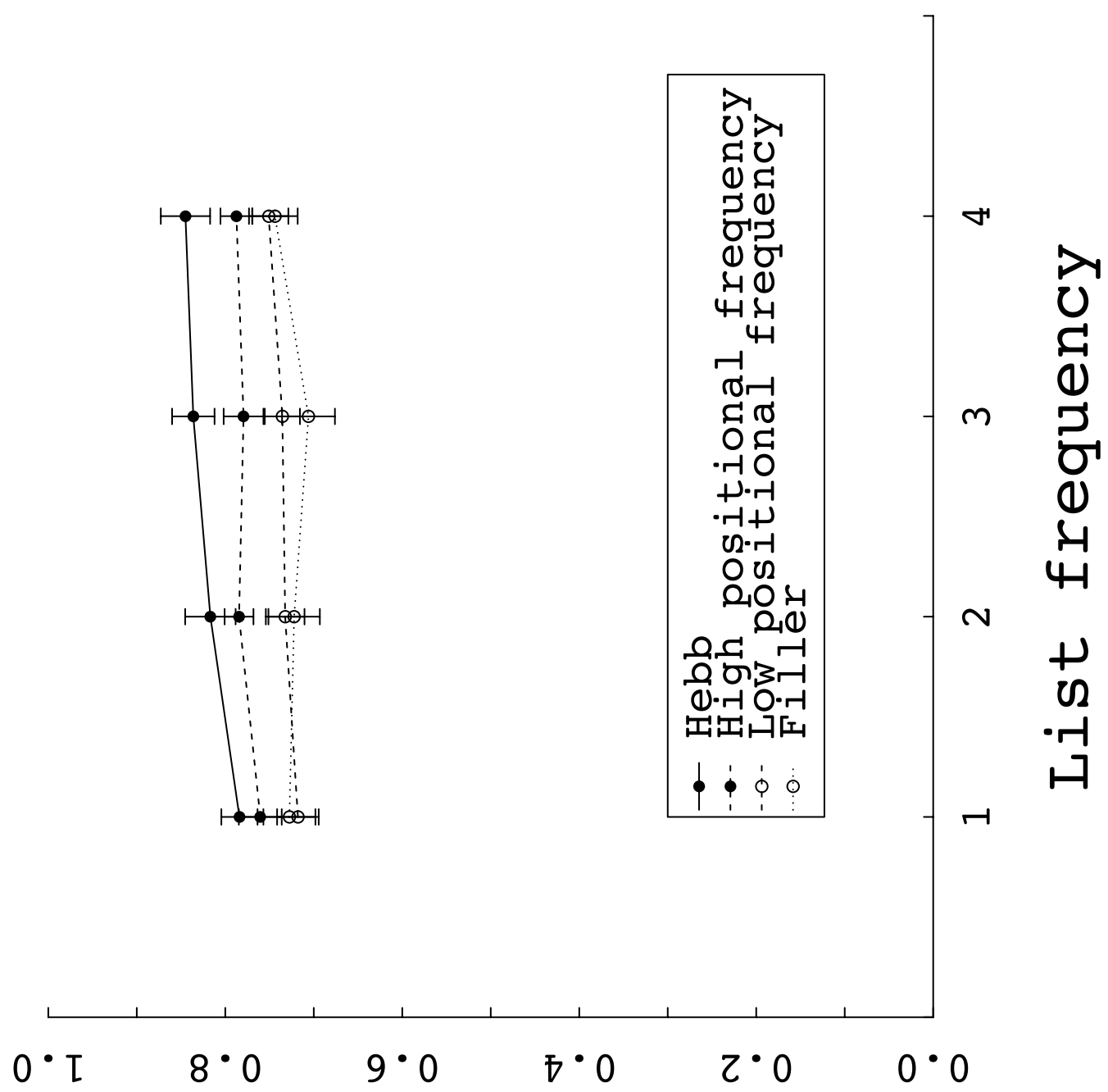

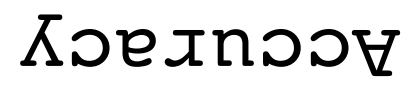

A 
Positional repetition in serial learning

52

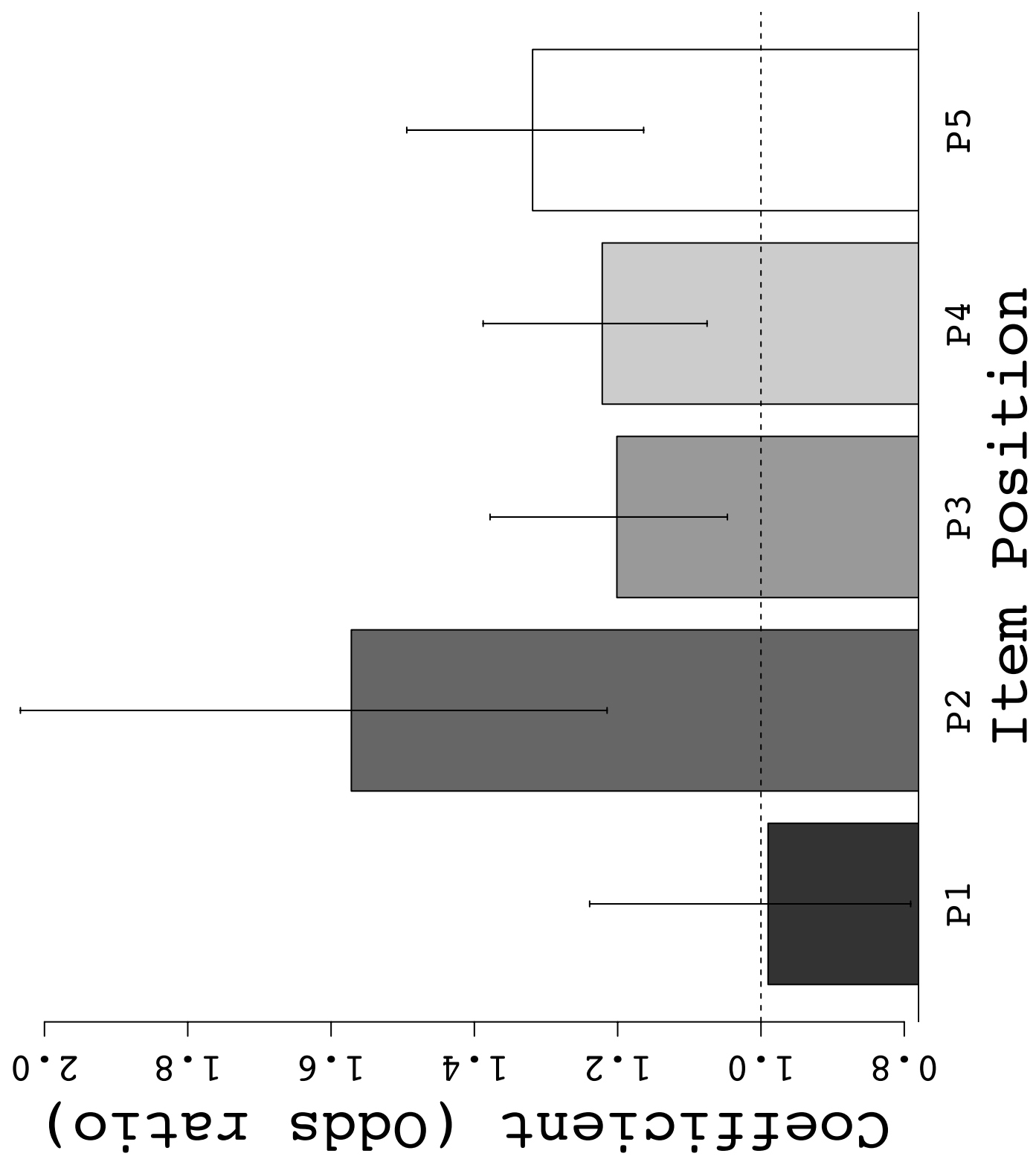

B

Figure 6. List frequency effects in Experiment 4 
Positional repetition in serial learning

53

\section{Results and Discussion}

Regression coefficients (odds ratios) of predictors are reported in Figures 5B and 6B (Detailed statistics are reported in Table A4).

Position-item frequency. For demonstrative purposes, we show in Figure 5A the recall accuracy as a function of positional frequency and trial number throughout the experiment. To make the figure intuitively understandable, we separated the mixed frequency lists into high and low position frequency items. Those items that were planned to be high and low positional frequency were respectively categorized as high- and low-positional frequency items. Across all positions, mean accuracy was calculated for each list/item type and trial number category (e.g., 1-32, 33-64). As positional frequency was manipulated and accumulated throughout the experiment, the positional frequency learning is reflected as difference in slopes for filled circles (i.e., Hebb and high-positional frequency) and unfilled circles (i.e., low-positional frequency and filler). Figure 5A indicated that positional frequency effect emerged after 64th trials. Highpositional frequency items were more accurately recalled than low-positional frequency items and items in filler lists, which consisted of low-positional frequency items. Since the accumulated positional frequency of the Hebb lists items was as high as high-positional frequency items in the mixed frequency lists, the positional frequency effects were also seen as the steeper slope of the Hebb list than low-positional frequency items. A formal regression analysis confirmed this pattern (Figure 5B, Table A4) where coefficients for positional frequency roughly correspond to difference in slopes for filled circles (i.e., Hebb and high-positional frequency) and unfilled circles (i.e., low-positional frequency and filler) in Figure 5A. Positionitem frequencies exhibited position selectivity significantly affecting the accuracy in 
Positional repetition in serial learning $\quad 54$

corresponding positions (e.g., P5F influenced accuracy in position $5 ; z \mathrm{~s}>2.17$ ). As the positional repetition frequency in each item in the Hebb list was completely equated with those in highpositional frequency items in the mixed lists, when the list frequency effects were regressed out, the positional frequency effect reflected a pure effect of positional repetition and could not be a byproduct of list repetition. The odds ratios for positional frequency ranged from 1.01 to 1.03 across positions (Figure 5B whose $\mathrm{y}$-axis is scaled for direct comparison with the list frequency effect in Figure 6B).

Although the above analyses indicate the presence of positional frequency effects, further confirmation is needed because the observed positional frequency effects might be confounded with the bi-item and/or tri-item frequency effects. As we presented the same position-item combinations through one block, same item-item combinations were also presented through the block if any two of adjacent items had high positional frequency (e.g., 03 in the first block of Table 3C). As we statistically controlled the positional frequencies in the other position than the tested position, confounding of these bi-item or tri-item frequency effects was supposed to be minimized in the above analysis. Nonetheless, to further confirm the presence of the pure positional frequency effects, we conducted follow-up regression analyses. In each position-wise analysis, those trials were excluded where to-be-tested and one or more adjacent item(s) had (nominal) high positional frequency. For example, trial 1 in Table 3 was excluded from the analyses of position 1-4 and trial 191 in Table 3 was included in the analyses of all positions. In the remaining trials, knowledge of bi-item/tri-item frequency cannot be utilized even if such knowledge could be acquired over the course of the experiment. Although the number of data points were reduced from 5701 in the main analysis, a fair number of data points remained (i.e., 3204-3563; more than one hundred trails per one participant on average). The same regression 
Positional repetition in serial learning $\quad 55$

analyses were conducted for these data sets except for the fact that list frequency related effects were not included. Note that any item in the Hebb list does not meet the inclusion criteria and that there was no variance in list frequency in the analyzed data sets. The detailed results of the regressions are shown in Table A5. Positional frequency effects were replicated in all positions $(z \mathrm{~S}>2.01)$ except for position $2(z=1.01)$. The lack of positional frequency effect in position 2 is attributable to the reduced number of data points and increased variance. The coefficients in terms of odds ratios (and log odds ratios) of position 2 frequency in the current and main analyses were $1.01(0.012)$ and $1.01(0.014)$, respectively. The 95\% confidence intervals for odds ratios (and log odds ratios) were 0.99-1.04 (-0.012-0.037) and 1.00-1.03 (0.001-0.026), respectively (Tables A4 and A5). It seems that the point estimation of the effect (i.e., coefficients) was not reduced but that reduction in the number of data points made the $95 \%$ confidence interval larger in the current analysis such that it fully covered the $95 \%$ confidence interval of the main analysis. If the confounding bi-item/tri-item frequency knowledge explained the presence of the positional frequency effect in the main analysis and the lack of it in the current analysis, then the effect should have been reduced in the current analysis. However, we did not find clear evidence for it. Taken together, these follow-up regression analyses confirm the presence of position-element association knowledge, though it is difficult to directly compare these positional frequency effects to the list frequency effects reported below.

List frequency. For demonstrative purposes, Figure 6A shows the recall accuracy as a function of list frequency, whose maximum was four and was reset at the beginning of each block. To make the comparison easy, lists/items that were presented in the same sub-block as Hebb lists were plotted in the same (dummy) frequency conditions as the Hebb list. As positional repetition frequency in the Hebb lists were identical to the high-positional frequency items in the 
Positional repetition in serial learning $\quad 56$

mixed lists, the difference in slopes of the Hebb list and high-positional frequency items indicates Hebb effect over and above the positional frequency effect. As can be seen in Figure 6A, the Hebb list showed a steeper slope compared to high-positional frequency items, indicating that the list repetition had an effect on recall accuracy over and above the positional frequency effects. Consistent with this visual inspection, formal regression analyses for each position revealed a significant effect for list repetition except for position 1 ( $z \mathrm{~s}>2.60$; Figure $6 \mathrm{~B}$, Table A4). When the positional frequency effects were regressed out, the list frequency effect reflected a pure effect of list repetition over and above the positional repetition effect. Although the Hebb effect was not observed in position 1, the coefficients for list frequency ranged from 1.20 to 1.57 across the other positions beyond the range of positional frequency (i.e., 1.01 to 1.03). In each of these positions, the $95 \%$ confidence interval for each coefficient for list frequency did not overlap with that for positional frequency (Figures 5B and 6B; Table A4). Taken together, the analyses indicate that list repetition effects emerge faster than positional repetition effects.

In sum, Experiment 4 replicated the gradually learned positional frequency effect. Direct comparison of effects of list repetition (i.e., the Hebb repetition) and positional repetition with completely equated repetition lag further confirmed that the positional frequency was learned more gradually than list frequency was. We also confirmed that the locus of learning is the phonological domain because the current experiment prevented participants from both spatial and motor learning by using random nonword-key mapping in each trial.

\section{General Discussion}


Positional repetition in serial learning

57

In previous research, position-element frequency effects on short-term retention of a sequence were shown with natural phonotactic frequency manipulation. However, it was unclear whether the acquisition mechanism of the position-element association knowledge was dissociable from that of sequence knowledge (i.e., the Hebb learning) and any other potentially confounding linguistic knowledge. On the other hand, the dissociation of the Hebb effect from subsequence knowledge has been shown without showing the position-element association knowledge itself. Several repetitions of the Hebb list resulted in a detectable increase in recall accuracy while the same number of positional repetitions showed little increase in accuracy, suggesting the dissociable learning of the former from the latter. Therefore, no study has shown the acquisition of position-element association knowledge dissociated from list repetition learning. Filling this gap, this study demonstrated successful learning of positional frequency in a laboratory learning paradigm. Three experiments manipulated to-be-learned positional frequency while other aspects of phonotactics were left random and/or controlled (i.e., list frequency manipulation). Although unexpected statistical regularity, by chance, might confound with the positional frequency within one of these randomly selected sets of to-be-learned structure, it is unlikely that it happened in all of the three sets of materials. The demonstrated positional frequency effect, therefore, should reflect learning of long-term position-element association. The dissociation of positional-element association learning and the Hebb learning was further shown by the difference in the learning rate. The emergence of the position-element frequency effect was more gradual than the Hebb list repetition effect. In the next sections, we will discuss some potential roles of these two types of learning.

Leaning systems with fast and slow learning rates have their own roles and collaborate to contribute to learning (Baddeley et al., 1998; McClelland, McNaughton, \& O’Reilly, 1995). Too 
Positional repetition in serial learning $\quad 58$

fast learning would cause catastrophic interference where learning of one thing overrides existing knowledge and eventually harms overall learning. Therefore, slow learning systems are adaptive in this respect especially to learning general patterns in the environment. However, the fast learning system that is relatively independent from existing knowledge is also important and adaptive. The environment, such as a linguistic environment, is not completely consistent and learners sometimes encounter a novel and structure incompatible stimulus (e.g., a specific word form might not have high phonotactic frequency); therefore, a fast learning system that is insensitive to existing knowledge is required. It seems adaptive that a system slowly learns position-element association knowledge, which is a form of knowledge of general patterns in linguistic environment, and that another system learns specific sequences such as word forms quickly. Actually, errors are also learned in Hebb learning that prevent perfect learning (Couture, Lafond, \& Tremblay, 2008). In a real linguistic environment, other types of knowledge, such as semantic knowledge, contribute to the reduction of initial errors and support successful learning (Jefferies, Bott, Ehsan, \& Lambon Ralph, 2011). It is inferred that phonological knowledge such as position-element association knowledge and/or a learning mechanism for it also contributes to language learning, compensating the Hebb learning.

Another compensatory role of long-term position-element association learning is inferred from a logical consequence of the nature of the linguistic environment. At the phonological level, language consists of essentially a closed set of elements. A closed set of phonemes is combined to create larger open sets of words, phrases, and so on. Hebb learning is ineffective for sequences that consist of a closed set of items especially when the repetition lag is longer. This is true probably because of the increase in chance of experiencing similar and interfering sequences (Melton, 1963; Page, Cumming, Norris, McNeil, \& Hitch, 2013; Page \& Norris, 2009). One 
Positional repetition in serial learning

59

compensatory form of learning and/or knowledge is subsequence level bi-element frequency learning. Chunking of two or more elements creates an open set at a subsequence/sublexical level and would avoid similarity based interference (Smalle et al., 2016). Position-element association learning/knowledge can be also compensating the lack of Hebb list learning. It is logically impossible that the positional repetition lag of a high-positional frequency item becomes longer in a closed set. In a closed set of $\mathrm{N}$ items, the minimum of the expected repetition lag of the high positional frequency is approximately $\mathrm{N}$ (i.e., even if the frequency is equally distributed, one of the items should repeat at least once in $\mathrm{N}$ times). Therefore, the more the set becomes closed, the less the (expected) repetition lag becomes. In this respect, the positional repetition learning can avoid the problem that the Hebb learning mechanism faces.

In conclusion, we have demonstrated gradual learning of long-term position-element association dissociating from Hebb list learning. Hebb learning has been shown to comprise a basis of acquisition of lexical knowledge (Mosse \& Jarrold, 2008; Szmalec et al., 2009, 2012). Given the importance of phonological knowledge in serial ordering (e.g., Dell et al., 2000; Gathercole et al., 1999; Gupta \& Tisdale, 2009; Hartley \& Houghton, 1996; Majerus et al., 2004; Nakayama et al., 2015; Tanida et al., 2015), it would be fruitful to further investigate the nature and functional role of position-element association learning in the context of language acquisition. 
Positional repetition in serial learning $\quad 60$

\section{Acknowledgements}

Part of the data and manuscript were submitted to the Graduate School of Education Kyoto University as a part of MN's doctoral thesis and were presented at the 31 st International Congress of Psychology. This research was financially supported in part by JSPS KAKENHI (Grant Number: 25380980) to SS. MN was supported by JSPS Postdoctoral Fellowships for Research Abroad. The funding source was not involved in study design, in the writing of the report, or in the decision to submit the article for publication. We thank Kaori Sakai and Yuki Tanida for assistance with stimuli creation and Satoru Nishiyama, Naoko Ohtsu, Kohei Morimoto, and Ayumi Tokoro for data collection. We are also grateful to Mike Page and two anonymous reviewers for helpful comments during the review process. We appreciate David Plaut for comments on the earlier version of the draft. 
Positional repetition in serial learning

61

\section{Appendix A}

\section{List of the stimuli}

Numbers indicate item number

\section{Experiment 1}

Learning lists

High frequency lists: 038247, 051389, 054719, 056284, 081429, 091547, 107593, 128659, 169754, 169854, 187249, 283169, 286439, 306728, 354729, 371254, 376159, 394208, 401735, 431968, 436287, 450719, 451237, 459786, 459812, 469182, 501284, 501928, 584932, 586721, 608721, 726389, 750238, 756198, 781609, 801325, 801459, 804759, 845231, 846251, 857419, $891704,905314,927638$

Low frequency lists: 037124, 046259, 056781, 057364, 095487, 095627, 159603, 180294, 195032, 201359, 249761, 263719, 296704, 305249, 321749, 351468, 401539, 401937, 419207, 451739, 459283, 497632, 507214, 512784, 517362, 581769, 586103, 614237, 621704, 651723, 674309, 681754, 689431, 716830, 758219, 786134, 798435, 846732, 856239, 864752, 874102, $926074,946703,961825$

Transfer lists

Day 2: 012798, 013298, 072536, 081793, 102836, 152794, 152896, 156079, 156230, 170235, 172584, 176039, 186094, 186274, 203578, 216794, 271835, 286573, 286793, 312094, 316285, 316795, 351096, 351720, 356824, 371024, 371856, 382094, 410286, 410835, 416273, 416573, 416829, 450876, 456289, 456879, 473528, 476895, 480529, 482093, 486035, 486253, 510289, 
Positional repetition in serial learning $\quad 62$

516298, 516784, 601794, 603594, 671598, 673259, 681054, 683759, 703526, 706835, 710295, 710594, 716095, 716534, 750834, 782035, 782590, 802796, 806735, 810573, 812796, 816024, 850294, 851024, 852739, 853279, 856793, 870234, 871529, 876254, 902538, 903874, 906874, 912584, 913876, 916285, 916873, 956730, 970825, 973284, 980526, 980724, 983720, 986274, 986753

Day 3: 012856, 016853, 051789, 053279, 053824, 071293, 071594, 073289, 076284, 076589, 082793, 083254, 086279, 086723, 102584, 103759, 106274, 106725, 176590, 182574, 183790, 203789, 210589, 213796, 213895, 216095, 250874, 251074, 256783, 281039, 310274, 312790, $316579,316875,356280,370856,371528,376584,376894,382590,403589,406895,412850$, 451836, 453089, 470235, 471026, 473826, 481053, 481270, 483096, 486573, 486725, 572094, 573086, 573284, 576238, 601293, 610298, 610584, 612895, 613250, 652870, 670285, 671098, 671238, 681574, 682039, 713259, 716258, 752830, 753086, 753298, 753896, 780296, 781296, 803274, 806574, 813796, 816279, 871234, 873059, 901854, 902856, 906258, 956024, 981736, 986530

\section{Experiment 2}

013829, 016489, 016532, 023597, 036478, 036549, 037265, 038769, 039851, 042518, 043286, 063298, 064172, 082539, 082961, 083457, 092476, 093672, 094172, 102548, 102973, 107253, 107548, 125073, 132609, 134568, 142578, 169478, 170529, 190487, 193487, 201478, 203459, 206381, 210479, 210754, 213069, 213460, 213546, 214568, 217469, 217569, 218970, 219067, 235479, 265097, 270569, 280579, 283145, 289476, 293468, 301462, 304689, 306842, 310769, 
Positional repetition in serial learning $\quad 63$

$312586,315987,317428,319450,319576,325468,342569,361572,362475,369408,384567$, 395168, 405673, 406173, 413269, 413908, 415867, 429076, 435872, 436798, 459178, 472198, 501469, 503218, 503748, 503928, 508629, 513067, 530869, 532741, 539461, 562847, 582379, $582439,602538,605431,610375,610578,613708,614973,617428,619250,623579,628479$, $632405,641705,642079,642508,643259,653478,682479,692481,692538,695178,702465$, 703492, 704368, 704598, 712435, 712598, 713428, 713529, 713846, 715069, 718369, 728561, 730568, 742593, 756390, 783429, 792506, 801539, 801796, 802479, 803419, 803579, 806329, 810973, 813679, 815074, 819456, 835471, 845061, 846501, 852471, 853109, 870263, 872439, 892071, 901468, 901768, 902435, 903472, 904573, 910426, 912576, 913486, 913574, 913768, 916705, 921460, 923450, 932564, 934028, 941568, 941672, 962514, 964872, 982130

\section{Experiment 3}

Repeated lists

043786, 305614, 402875, 451078, 468097, 514378, 821043, 845796, 859710, 901435, 931567, 958310

Non-repeated lists

017356, 043697, 048697, 054817, 064378, 087962, 089642, 138604, 140875, 148362, 150246, 158024, 160584, 164539, 165094, 167805, 167923, 169058, 174265, 204859, 215870, 250397, 250937, 259036, 271803, 278940, 279365, 305487, 308154, 314026, 321496, 325401, 325867, 325908, 329410, 347561, 347962, 358947, 359860, 364920, 365471, 390251, 392875, 395417, 407283, 432576, 450678, 461750, 462753, 475891, 486305, 493587, 518306, 520376, 529380, 
Positional repetition in serial learning $\quad 64$

531279, 538406, 546708, 567401, 572946, 584621, 590312, 593178, 608134, 625013, 634718, 641893, 642891, 659142, 682504, 691045, 693548, 720985, 739581, 756381, 765208, 795068, 798062, 823516, 826579, 834602, 850623, 862594, 863049, 865974, 867903, 869514, 875290, $893142,923071,924830,938526,942638,951083,957148,965078$

\section{Experiment 4}

This list also exemplifies a presentation order of the entire experiment. In each sub-block of four list, first and third list were mixed positional frequency lists $(*)$, second list was a Hebb list $(+)$, which is repeated four times, and fourth list was low frequency or filler list (\#).

$$
\begin{aligned}
& 03492^{*}, 12568+, 54879^{*}, 46780 \#, 03415^{*}, 12568+, 68379^{*}, 79043 \#, 03456^{*}, 12568+, 30179^{*}, \\
& 97204 \#, 03421^{*}, 12568+, 85679^{*}, 21937 \#, 34569^{*}, 02478+, 13652^{*}, 69321 \#, 28569^{*}, 02478+, \\
& 13704^{*}, 86143 \#, 70569^{*}, 02478+, 13286^{*}, 95017 \#, 47569^{*}, 02478+, 13895^{*}, 51930 \#, 37498^{*}, \\
& 03569+, 12876^{*}, 74280 \#, 29418^{*}, 03569+, 12375^{*}, 48027 \#, 91408^{*}, 03569+, 12673^{*}, 60152 \#, \\
& 56438^{*}, 03569+, 12974^{*}, 85741 \#, 53168^{*}, 02479+, 14537^{*}, 61825 \#, 73968^{*}, 02479+, 18546^{*}, \\
& 37610 \#, 93068^{*}, 02479+, 16584^{*}, 85201 \#, 23768^{*}, 02479+, 10593^{*}, 49352 \#, 05428^{*}, 13569+, \\
& 62371^{*}, 74986 \#, 01498^{*}, 13569+, 82074^{*}, 98207 \#, 07418^{*}, 13569+, 32675^{*}, 26753 \#, 09438^{*}, \\
& 13569+, 42170^{*}, 50842 \#, 04769^{*}, 12578+, 93450^{*}, 46023 \#, 07869^{*}, 12578+, 63412^{*}, 59687 \#, \\
& 05269^{*}, 12578^{*}, 73406^{*}, 80134 \#, 01369^{*}, 12578+, 23495^{*}, 38941 \#, 23176^{*}, 02469+, 17598^{*}, \\
& 96087 \#, 83675^{*}, 02469+, 14528^{*}, 78354 \#, 53271^{*}, 02469+, 10538^{*}, 65913 \#, 43872^{*}, 02469+, \\
& 19508^{*}, 31740 \#, 67528^{*}, 13469+, 02175^{*}, 98013 \#, 30598^{*}, 13469+, 02874^{*}, 81257 \#, 29508^{*},
\end{aligned}
$$


Positional repetition in serial learning $\quad 65$

13469+, 02376*, 54632\#, 76548*, 13469+, 02971*, 45780\#, 04189*, 13568+, 32476*, 49302\#, 08719*, 13568+, 62475*, 75924\#, 06259*, 13568+, 92473*, 87091\#, 01649*, 13568+, 52470*, 20837\#, 13854*, 02569+, 29478*, 95083\#, 13295*, 02569+, 61478*, 74321\#, 13746*, 02569+, 30478*, 87130\#, 13907*, 02569+, 56478*, 48612\#, 31279*, 03568+, 12405*, 67091\#, 24879*, 03568+, 12430*, 75943\#, 40179*, 03568+, 12486*, 59724\#, 86379*, 03568+, 12457*, 98612\#, 72408*, 13579+, 04261*, 26035\#, 52418*, 13579+, 09867*, 98320\#, 62458*, 13579+, 07964*, 80746\#, 32498*, 13579+, 05162*, 41683\# 
Positional repetition in serial learning $\quad 66$

\section{Appendix B}

Table A1. Results of logistic regressions in Experiment 1

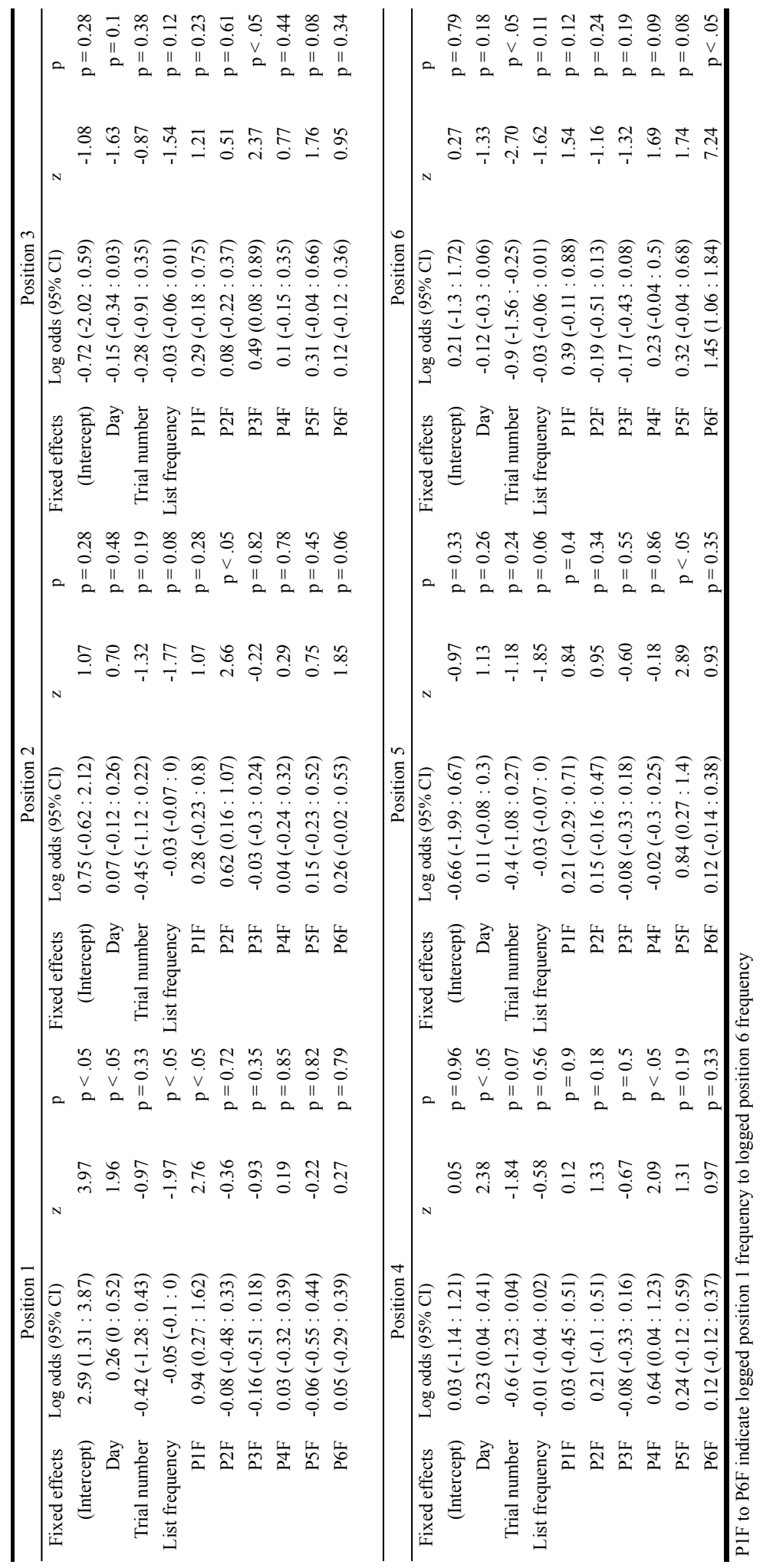


Positional repetition in serial learning

67

Table A2. Results of logistic regressions in Experiment 2

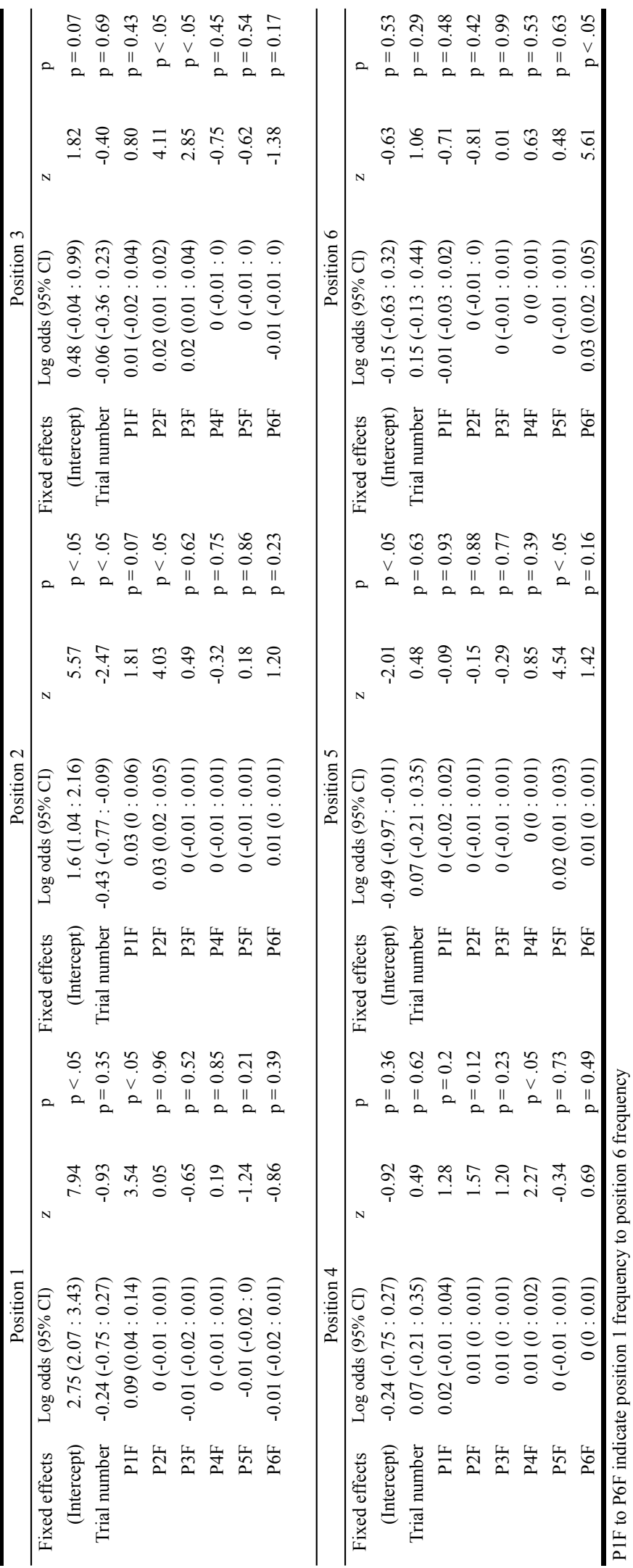


Positional repetition in serial learning

68

Table A3. Results of logistic regressions in Experiment 3

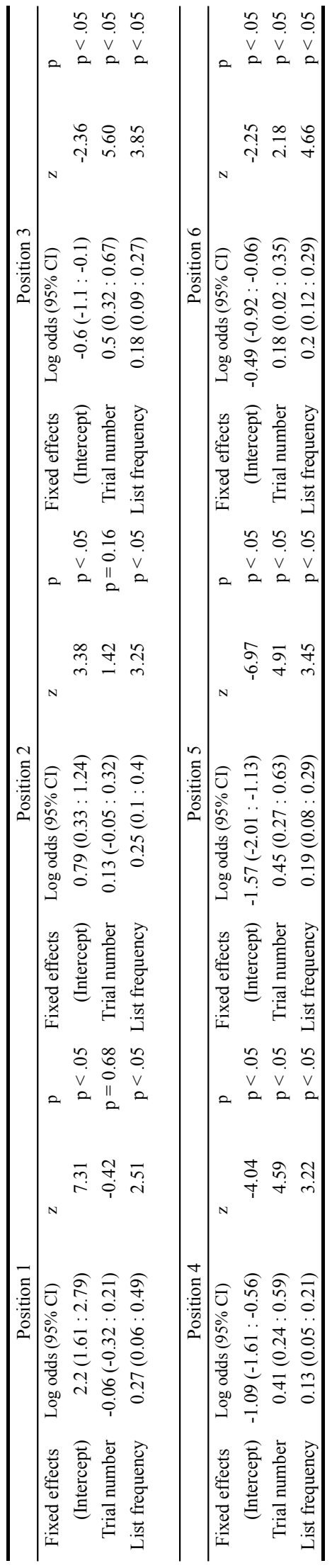


Positional repetition in serial learning

69

Table A4. Results of the main logistic regressions in Experiment 4

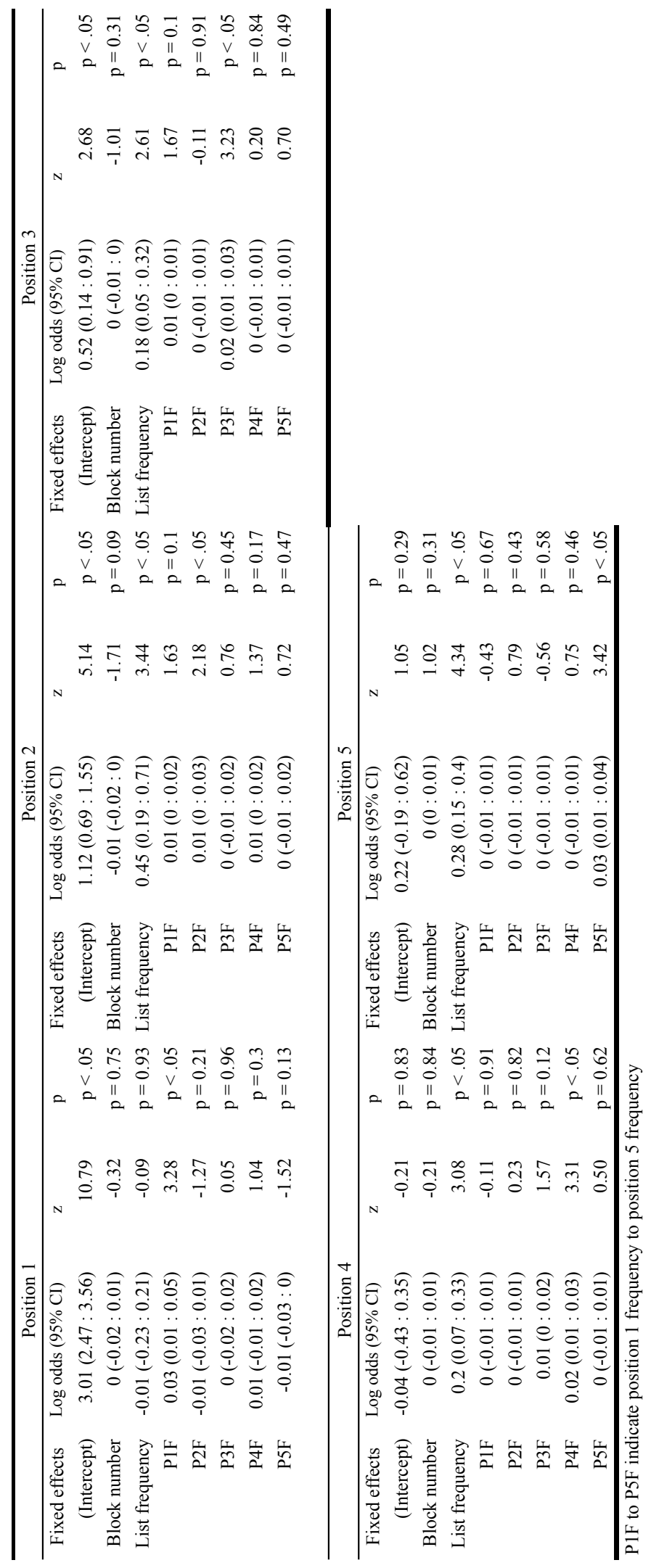


Positional repetition in serial learning $\quad 70$

Table A5. Results of the follow-up logistic regressions in Experiment 4

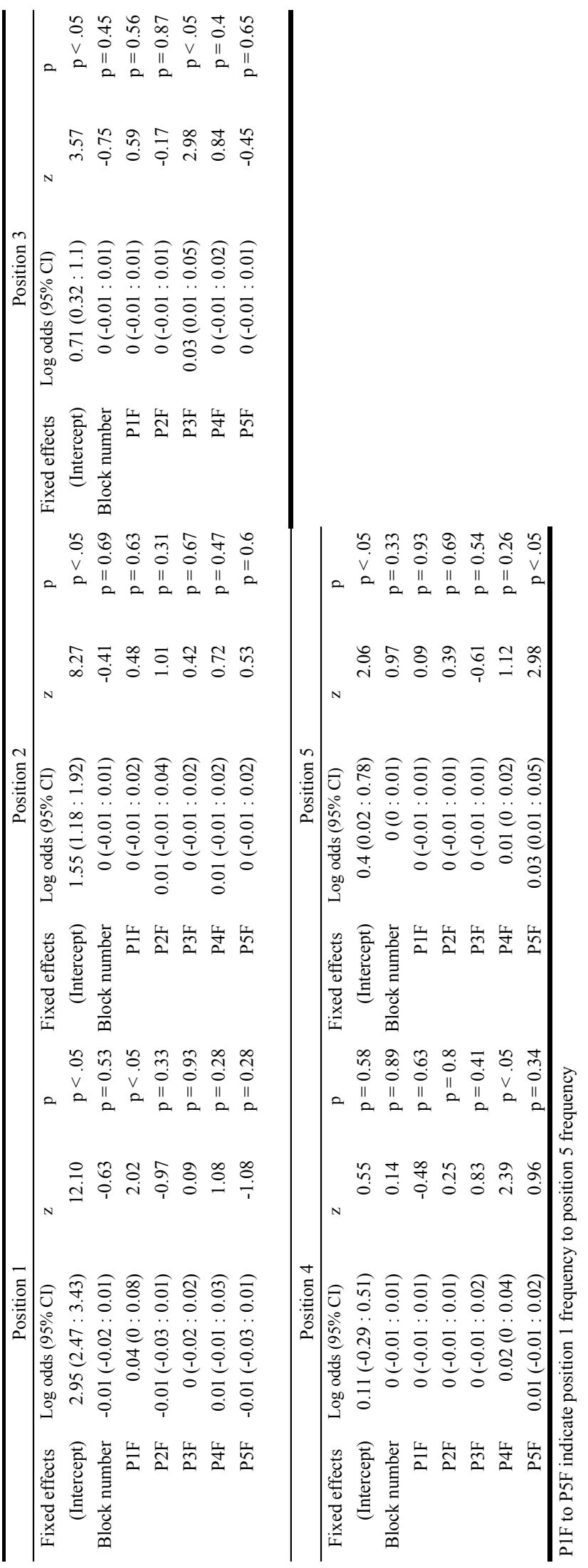




\section{References}

Amano, S., \& Kondo, T. (2000). Japanese NTT database series: Lexical properties of Japanese, word-frequency (II). Tokyo: Sanseido [in Japanese].

Armstrong, B. C., Watson, C. E., \& Plaut, D. C. (2012). SOS! An algorithm and software for the stochastic optimization of stimuli. Behavior Research Methods, 44(3), 675-705. http://doi.org/10.3758/s13428-011-0182-9

Baddeley, A. D., Gathercole, S. E., \& Papagno, C. (1998). The phonological loop as a language learning device. Psychological Review, 105(1), 158-73. Retrieved from http://www.ncbi.nlm.nih.gov/pubmed/9450375

Botvinick, M. M. (2005). Effects of domain-specific knowledge on memory for serial order. Cognition, 97(2), 135-51. http://doi.org/10.1016/j.cognition.2004.09.007

Botvinick, M. M., \& Bylsma, L. M. (2005). Regularization in short-term memory for serial order. Journal of Experimental Psychology. Learning, Memory, and Cognition, 31(2), 3518. http://doi.org/10.1037/0278-7393.31.2.351

Botvinick, M. M., \& Plaut, D. C. (2006). Short-term memory for serial order: a recurrent neural network model. Psychological Review, 113(2), 201-233. http://doi.org/10.1037/0033295X.113.2.201

Brown, G. D. A., Preece, T., \& Hulme, C. (2000). Oscillator-based memory for serial order. Psychological Review, 107(1), 127-181. http://doi.org/10.1037//0033-295X.107.1.127

Burgess, N., \& Hitch, G. J. (2006). A revised model of short-term memory and long-term learning of verbal sequences. Journal of Memory and Language, 55(4), 627-652. http://doi.org/10.1016/j.jml.2006.08.005

Cousineau, D. (2005). Confidence intervals in within-subject designs: A simpler solution to Loftus and Masson's method. Tutorials in Quantitative Methods for Psychology, 1(1), 4245.

Couture, M., Lafond, D., \& Tremblay, S. (2008). Learning correct responses and errors in the Hebb repetition effect: two faces of the same coin. Journal of Experimental Psychology. Learning, Memory, and Cognition, 34(3), 524-32. http://doi.org/10.1037/02787393.34.3.524

Cumming, N., Page, M. P. A., \& Norris, D. (2003). Testing a positional model of the Hebb effect. Memory, 11(1), 37-41. http://doi.org/10.1080/09658210143000452

Dell, G. S., Reed, K. D., Adams, D. R., \& Meyer, A. S. (2000). Speech errors, phonotactic constraints, and implicit learning: a study of the role of experience in language production. Journal of Experimental Psychology: Learning, Memory, and Cognition, 26(6), 1355-1367. Retrieved from http://psycnet.apa.org/journals/xlm/26/6/1355/

Farrell, S. (2012). Temporal clustering and sequencing in short-term memory and episodic memory. Psychological Review, 119(2), 223-71. http://doi.org/10.1037/a0027371

Fischer-Baum, S., \& McCloskey, M. (2015). Representation of item position in immediate serial recall : Evidence from intrusion errors. Journal of Experimental Psychology: Learning, Memory, and Cognition. http://doi.org/http://dx.doi.org/10.1037/xlm0000102

Gathercole, S. E., Frankish, C. R., Pickering, S. J., \& Peaker, S. (1999). Phonotactic Influences on Short-Term Memory. Journal of Experimental Psychology: Learning, Memory, and Cognition, 25(1), 84-95. Retrieved from http://psycnet.apa.org/journals/xlm/25/1/84/ 
Gupta, P., \& Tisdale, J. (2009). Does phonological short-term memory causally determine vocabulary learning? Toward a computational resolution of the debate. Journal of Memory and Language, 61(4), 481-502. http://doi.org/10.1016/j.jml.2009.08.001

Hartley, T., \& Houghton, G. (1996). A linguistically constrained model of short-term memory for nonwords. Journal of Memory and Language, 35, 1-31. Retrieved from http://wwwusers.york.ac.uk/ th512/papers/HartleyJml1996.pdf

Hebb, D. O. (1961). Distinctive features of learning in the higher animal. In J. F. Delafresnaye (Ed.), Brain mechanisms and learning (pp. 37-46). New York: Oxford University Press.

Henson, R. N. A. (1998). Short-term memory for serial order: the Start-End Model. Cognitive Psychology, 36(2), 73-137. http://doi.org/10.1006/cogp.1998.0685

Henson, R. N. A. (1999). Positional information in short-term memory : Relative or absolute? Memory \& Cognition, 27(5), 915-927. Retrieved from http://link.springer.com/article/10.3758/BF03198544

Henson, R. N. A., \& Burgess, N. (1997). Representations of serial order. In J. Bullinaria, D. Glasspool, \& G. Houghton (Eds.), Proceedings of fourth neural computation and psychology workshop. (pp. 283-300). London: Springer.

Hitch, G. J., Fastame, M. C., \& Flude, B. (2005). How is the serial order of a verbal sequence coded? Some comparisons between models. Memory, 13(3-4), 247-258. http://doi.org/10.1080/09658210344000314

Hurlstone, M., Hitch, G., \& Baddeley, A. (2014). Memory for serial order across domains: An overview of the literature and directions for future research. Psychological Bulletin, 140(2), 339-373. http://doi.org/10.1037/a0034221

Jaeger, T. F. (2008). Categorical Data Analysis: Away from ANOVAs (transformation or not) and towards Logit Mixed Models. Journal of Memory and Language, 59(4), 434-446. http://doi.org/10.1016/j.jml.2007.11.007

Jefferies, E., Bott, S., Ehsan, S., \& Lambon Ralph, M. A. (2011). Phonological learning in semantic dementia. Neuropsychologia, 49(5), 1208-18. http://doi.org/10.1016/j.neuropsychologia.2011.01.036

Lashley, K. (1951). The problem of serial order in behavior. In L. A. Jeffress (Ed.), Cerebral Mechanisms in Behaviour: the Hixon Symposium (pp. 112-136). New York: Wiley.

Majerus, S., Linden, M. Van Der, Mulder, L., Meulemans, T., \& Peters, F. (2004). Verbal shortterm memory reflects the sublexical organization of the phonological language network: Evidence from an incidental phonotactic learning paradigm. Journal of Memory and Language, 51(2), 297-306. http://doi.org/10.1016/j.jml.2004.05.002

Majerus, S., Martinez Perez, T., \& Oberauer, K. (2012). Two distinct origins of long-term learning effects in verbal short-term memory. Journal of Memory and Language, 66(1), 3851. http://doi.org/10.1016/j.jml.2011.07.006

McClelland, J. L., McNaughton, B. L., \& O'Reilly, R. C. (1995). Why there are complementary learning systems in the hippocampus and neocortex: Insights from the successes and failures of connectionist models of learning and memory. Psychological Review, 102(3), 419-457. Retrieved from http://psycnet.apa.org/psycinfo/1995-42327-001

Melton, A. W. (1963). Implications of short-term memory for a general theory of memory. Journal of Verbal Learning and Verbal Behavior, 2(1), 1-21. http://doi.org/10.1016/S00225371(63)80063-8 
Mosse, E. K., \& Jarrold, C. (2008). Hebb learning, verbal short-term memory, and the acquisition of phonological forms in children. Quarterly Journal of Experimental Psychology (2006), 61(4), 505-14. http://doi.org/10.1080/17470210701680779

Nakayama, M., Tanida, Y., \& Saito, S. (2015). Long-term phonological knowledge supports serial ordering in working memory. Journal of Experimental Psychology: Learning, Memory and Cognition, 41(5), 1570-1578. http://doi.org/http://dx.doi.org/10.1037/a0038825

O'Reilly, R. C., \& Soto, R. (2001). A model of the phonological loop: Generalization and binding. In T. G. Dietterich, S. Becker, \& Z. Ghahramani (Eds.), Advances in neural information processing systems.

Page, M. P. A., Cumming, N., Norris, D., McNeil, A. M., \& Hitch, G. J. (2013). Repetitionspacing and item-overlap effects in the Hebb repetition task. Journal of Memory and Language, 69(4), 506-526. http://doi.org/10.1016/j.jml.2013.07.001

Page, M. P. A., \& Norris, D. G. (2009). A model linking immediate serial recall, the Hebb repetition effect and the learning of phonological word forms. Philosophical Transactions of the Royal Society of London. Series B, Biological Sciences, 364(1536), 3737-53. http://doi.org/10.1098/rstb.2009.0173

R Core Team. (2013). R: A language and environment for statistical computing. Vienna, Austria: R Foundation for Statistical Computing. Retrieved from http://www.r-project.org/

Schwartz, M., \& Bryden, M. P. (1971). Coding factors in the learning of repeated digit sequences, 87(3), 331-334.

Smalle, E. H. M., Bogaerts, L., Simonis, M., Duyck, W., Page, M. P. A., Edwards, M. G., \& Szmalec, A. (2016). Can Chunk Size Differences Explain Developmental Changes in Lexical Learning? Frontiers in Psychology, 6(January), 1-14. http://doi.org/10.3389/fpsyg.2015.01925

Szmalec, A., Duyck, W., Vandierendonck, A., Mata, A. B., \& Page, M. P. A. (2009). The Hebb repetition effect as a laboratory analogue of novel word learning. Quarterly Journal of Experimental Psychology, 62(3), 435-43. http://doi.org/10.1080/17470210802386375

Szmalec, A., Page, M. P. A., \& Duyck, W. (2012). The development of long-term lexical representations through Hebb repetition learning. Journal of Memory and Language, 67(3), 342-354. http://doi.org/10.1016/j.jml.2012.07.001

Tanida, Y., Ueno, T., Lambon Ralph, M. A., \& Saito, S. (2015). The roles of long-term phonotactic and lexical prosodic knowledge in phonological short-term memory. Memory \& Cognition, 43(3), 500-519. http://doi.org/10.3758/s13421-014-0482-2

van Casteren, M., \& Davis, M. H. (2006). Mix, a program for pseudorandomization. Behavior Research Methods, 38(4), 584-589.

Warker, J. A., \& Dell, G. S. (2006). Speech Errors Reflect Newly Learned Phonotactic Constraints. Journal of Experimental Psychology: Learning, Memory, and Cognition, 32(2), 387-398. http://doi.org/10.1037/0278-7393.32.2.387 
Positional repetition in serial learning

74

\section{Figure captions}

Figure 1. Position-item frequency effects in Experiment 1

A: Recall accuracy as a function of trial number and positional frequency. Error bars indicate within-participant $95 \%$ confidence intervals (Cousineau, 2005) based on raw recall accuracy.

B: Regression coefficients of position-item frequencies. Odds ratios and $95 \%$ confidence intervals are displayed. Darker bars indicate effects of position-item frequency for earlier positions.

Figure 2. List frequency effects in Experiment 1

A: Recall accuracy as a function of list frequency and day. Each dot is plotted to roughly correspond to the presented timing for each day. Error bars indicate within-participant $95 \%$ confidence intervals (Cousineau, 2005) based on raw recall accuracy.

B: Regression coefficients of list frequencies. Odds ratios and $95 \%$ confidence intervals are displayed.

Figure 3. Position-item frequency effects in Experiment 2

A: Recall accuracy as a function of trial number and positional frequency. Error bars indicate within-participant $95 \%$ confidence intervals (Cousineau, 2005) based on raw recall accuracy.

B: Regression coefficients of position-item frequencies. Odds ratios and $95 \%$ confidence intervals are displayed. Darker bars indicate effects of position-item frequency for earlier positions.

Figure 4. List frequency effects in Experiment 3

A: Recall accuracy as a function of list frequency. Solid lines indicate Hebb lists, and dashed lines indicate non-repeated filler lists that are presented before and after the corresponding Hebb lists (see main text). Error bars indicate within-participant $95 \%$ confidence intervals (Cousineau, 2005) based on raw recall accuracy. 
Positional repetition in serial learning

75

B: Regression coefficients of list frequencies. Odds ratios and $95 \%$ confidence intervals are displayed.

Figure 5. Position-item frequency effects in Experiment 4

A: Recall accuracy as a function of trial number and list type. Error bars indicate withinparticipant $95 \%$ confidence intervals (Cousineau, 2005) based on raw recall accuracy.

B: Regression coefficients of position-item frequencies. Odds ratios and $95 \%$ confidence intervals are displayed. Darker bars indicate effects of position-item frequency for earlier positions.

Figure 6. List frequency effects in Experiment 4

A: Recall accuracy as a function of list frequency. Error bars indicate within-participant $95 \%$ confidence intervals (Cousineau, 2005) based on raw recall accuracy.

B: Regression coefficients of list frequencies. Odds ratios and $95 \%$ confidence intervals are displayed. 
Positional repetition in serial learning

76

\section{Table notes}

Table 1. Artificial phonotactics in Experiment 1

Table 2. Artificial phonotactics in Experiment 2

Table 3. Artificial phonotactics in Experiment 4 